\title{
Orexin-A Alleviates Astrocytic Apoptosis and Inflammation via Inhibiting OX1R-mediated NF-KB and MAPK Signaling Pathways in Cerebral Ischemia/reperfusion Injury
}

\section{Dandan Xu}

Neurobiology Key Laboratory of Jining Medical University

\section{Tingting Kong}

Caoxian People's Hospital

\section{Ziqi Shao}

Neurobiology Key Laboratory of Jining Medical University

Rumin Zhang

Neurobiology Key Laboratory of Jining Medical University

Shengnan Zhang

Neurobiology Key Laboratory of Jining Medical University

Qingxia Kong

Affiliated Hospital of Jining Medical University

Jing Chen

Warkwick Medical School, University of Warwick

\section{Baohua Cheng}

Neurobiology Key Laboratory of Jining Medical University

Chunmei Wang ( $\nabla$ wangchunmei410@mail.jnmc.edu.cn )

Jining Medical University

\section{Research}

Keywords: Orexin-A (OXA), nuclear factor-Kb (NF-KB), astrocyte, inflammation, mitogen-activated protein kinase (MAPK), cerebral ischemia/reperfusion injury (CIRI), apoptosis

Posted Date: January 22nd, 2021

DOl: https://doi.org/10.21203/rs.3.rs-151920/v1

License: (c) (i) This work is licensed under a Creative Commons Attribution 4.0 International License. Read Full License 


\section{Abstract}

Background: Orexin-A (OXA) is a neuropeptide with neuroprotective effect by reducing cerebral ischemia/reperfusion injury (CIRI). Inflammation and apoptosis mediated by astrocyte activation are the key pathological mechanisms for CIRI. We thus attempted to confirm neuroprotective effects of OXA on astrocytic inflammation and apoptosis in CIRI and clarify the relative mechanisms.

Methods: A middle cerebral artery occlusion and reperfusion (MCAO/R) rat model and U251 glioma cells model subjected to oxygen glucose deprivation and reperfusion (OGD/R) were established, with or without OXA treatment. Neurological deficit score was determined, and cerebral infarct volume was evaluated by 2,3,5-triphenyltetrazolium chloride (TTC) staining. Western Blot was used to detect the expressions of NF-KB p65, p-ERK, p-p38, GFAP, OX1R, IL-1 $\beta$, TNF-a, IL-6, iNOS, Bcl-2, Bax, CytC, cleaved caspase-9 and cleaved caspase-3 in vivo and vitro. Pro-inflammatory cytokines in cell supernatant IL-1 $\beta$, TNF- $a$ and IL-6 were determined by ELISA. Hoechst 33342 staining was used to detect the apoptosis of astrocyte. Immunofluorescent staining was performed to assess the nuclear translocation of p65 and the expression of GFAP.

Results: The results showed that OXA significantly improved neurological deficit score and decreased the volume of infarct area in brain. OXA decreased inflammatory mediators, inhibited astrocyte activation and nuclear translocation of NF-KB and phosphorylation of MAPK/ERK and MAPK/p38. Besides, OXA suppressed apoptosis via upregulating the ratio of $\mathrm{Bcl}-2 / \mathrm{Bax}$ and downregulating cytochrome $\mathrm{C}$, cleavedcaspase-9 and cleaved caspase-3.

Conclusions: Overall, it was concluded that OXA exerts neuroprotective effect during CIRI through attenuating astrocytes apoptosis, astrocytes activation and pro-inflammatory cytokines production, by Inhibiting OX1R-mediated NF-KB, MAPK/ERK and MAPK/p38 signaling pathways. The progress in our study are helpful to elucidate the molecular mechanisms of OXA neuroprotection, which could lead to the development of new treatment strategies for ischemic stroke.

\section{Background}

Acute ischemic stroke (AIS) is the main cause of disability and is one of the leading causes of adult death worldwide [1]. At present, mechanical thrombectomy and thrombolysis with recombinant tissue plasminogen activator (rtPA) are the preferred treatments for patients without contraindications to systemic thrombolysis within the $4.5 \mathrm{~h}$ time-window. So timely successful reperfusion is the most effective treatment for patients with AIS [2]. However, restoration of blood supply can further aggravate brain tissue damage due to ischemia/reperfusion (I/R) injury [3]. Cerebral I/R injury involves a series of pathophysiological injury mechanisms, including excitotoxicity, oxidative stress, inflammation and apoptosis. The inflammatory response is an essential step in the occurrence and development of ischemic brain injury $[4,5]$. 
Astrocytes are the most abundant glial cell types in the brain and make up about $50 \%$ of human brain volume [6]. They provide metabolic and trophic support to neurons and modulate synaptic activity under normal physiological conditions. Moreover, the considerable evidences prove that astrocytes play a neuroprotective role through the antioxidant defense, metabolic support, the secretion of neuroprotective substances, and astrocyte-neuron interaction. In general, astrocytes lead to the neuroprotection and survival of neurons: any astrocyte dysfunction will seriously affect the survival of neurons [7]. Accordingly, considering the existence of astrocyte apoptosis in the ischemic damaged brain [8], it is particularly important to study the anti-apoptotic drugs in astrocytes and the precise molecular mechanism of the drugs.

Astrocyte-mediated inflammatory response in stroke is worthy of attention [9]. Various cytokines, such as interleukin IL-6 and IL-1 $\beta$, are released from necrotic neurons due to immune response after stroke [10]. These cytokines cause the activation and aggregation of astrocytes [11] and activated astrocytes will produce more pro-inflammatory factors, including IL-1 $\beta$ and tumor necrosis factor- $a$ (TNF- $a$ ), which further aggravate the ischemic damage [12]. Accumulating evidence suggests that astrocyte-mediated inflammatory response can cause secondary damage after cerebral I/R $[12,13]$. Therefore, targeting astrocytic inflammation may provide novel therapeutic strategies for neuroprotection after stroke.

Research reports that the Nuclear factor kappa light-chain enhancer of activated B cells (NF-KB) signaling pathway [14] and mitogen-activated protein kinase (MAPK) signaling pathway are the main pathways of inflammation regulation [15]. NF-KB plays an important role in the pathological process of ischemic stroke, which can be activated by hypoxia, reactive oxygen species (ROS), and increased inflammatory mediators during reperfusion [16]. The p65/p50 subunit is the most common heterodimer of NF-KB complex, and p65 is the most important due to its multifunctional domain [17]. The activated p65 translocates to the nucleus, leading to the expression of downstream pro-inflammatory genes, including cyclooxygenase (COX)-2, inducible nitric oxide synthase (iNOS), IL-1ß, IL-6, and so on $[18,19]$. The MAPKs in mammals include c-Jun N-terminal kinases (JNKs), p38 MAPK, and extracellular signal-regulated kinase (ERK) [20]. It is reported that the MAPK signaling pathway is involved in various cellular activities including proliferation, differentiation, apoptosis or survival, inflammation, and innate immunity. Moreover, the p38 MAPK pathway leads to inflammation mediated by astrocytes and microglia [21]. Therefore, regulating inflammation-related pathways MAPK pathway and NF-KB pathway may be beneficial to neuroprotection after stroke.

Orexin-A (OXA) and Orexin-B (OXB) are hypothalamic neuropeptides [22], which are involved in a variety of pathophysiological processes, including the regulation of the sleep/wakefulnessstate, energy homeostasis, reward seeking, and drug addiction [23, 24]. Furthermore OXA and OXB exert their effects by binding two G-protein-coupled receptors (GPCRs), orexin receptor type 1 (OX1R) and orexin receptor type 2 (OX2R) [25]. In particular, there is evidence that the binding affinity of OXA to OXIR is 5-100 times that of $O X 2 R$, while the binding affinity of OXA and OXB to OX2R is similar [26]. OXA has been reported to have neuroprotective effects [27], and our laboratory confirmed that OXA can reduce cerebral ischemiareperfusion injury in rats [28]. Numerous evidences strongly suggest that OXA can orchestrate its anti- 
inflammatory functions not only in central nervous system (CNS), but also in periphery [27, 29, 30]. However, the anti-inflammatory effects and molecular mechanisms of OXA in cerebral I/R have not been fully studied.

Since inflammation is the most important mechanism involved in cerebral I/R injury, in this study we explored the anti-inflammatory effects of OXA in vitro and in vivo and the corresponding molecular mechanisms. On the other hand, OXA reduces cerebral I/R injury by inhibiting neuronal apoptosis, which has been confirmed in our previous studies [31]. However, whether OXA can reduce cerebral I/R injury by inhibiting astrocyte apoptosis has not yet been elucidated, so we also explored the effect of OXA on astrocyte apoptosis. In short, the progress in the neuroprotective mechanism of OXA may provide new therapeutic strategies and molecular targets for ischemic stroke.

\section{Materials And Methods}

\subsection{Animals}

Male Sprague-Dawley (SD) rats, aged 8-9 weeks (weighing $250 \pm 10 \mathrm{~g}$ ), were purchased from Pengyue Experimental Animal Co., Ltd. (Jinan, China). All rats were acclimated for 1 week in a temperature of 24$26^{\circ} \mathrm{C}$ for $12 \mathrm{~h}$ light/dark cycles with sufficient food and water prior to the experiment. All animal experiments were conducted following the National Experimental Animal Feeding Guidelines and were approved by the Animal Ethics Committee of Jining Medical University.

\subsection{Middle cerebral artery occlusion (MCAO) model}

First, the rats were fasted for $12 \mathrm{~h}$ before the operation. After that, the rats were anesthetized by intraperitoneal injection of $10 \%$ chloral hydrate $(300 \mathrm{mg} / \mathrm{kg})$. Then, the rat was fixed in the supine position, and the right common carotid artery, external carotid artery (ECA), and internal carotid artery (ICA) were exposed and separated by median jugular incision. A 2.5 nylon mono-filament was inserted through the common carotid artery into the lumen of the ICA until it blocked the origin of the right middle cerebral artery. The filament was subsequently removed to allow reperfusion for $24 \mathrm{~h}$ after occlusion for 2 h. The sham group was treated the same as the model group except for the occlusion of the middle cerebral arteries.

The rats were randomly allocated to the four groups: sham treatment with vehicle (Sham group), sham treatment with OXA (OXA group), MCAO-reperfusion with vehicle (MCAO/R group), and MCAO/R with intracerebroventricular injection of OXA (MCAO/R + OXA group). A flow chart of the in vivo study design is shown in Fig. 1.

\subsection{Intracerebroventricular (ICV) injection}

At the beginning of reperfusion, the rats were anesthetized using $10 \%$ chloral hydrate $(300 \mathrm{mg} / \mathrm{kg})$ via intraperitoneal injection. A burr hole was carefully made in the skull at $0.8 \mathrm{~mm}$ dorsal and $1.6 \mathrm{~mm}$ lateral 
to the right from bregma using a Dremel drill. Then, $10 \mu \mathrm{L}$ OXA (30 $\mu \mathrm{g} / \mathrm{kg}$; Phoenix Pharmaceuticals, St. Joseph, MO, USA) [32] or $10 \mu \mathrm{L}$ vehicle $(0.9 \% \mathrm{NaCl})$ were injected at $2 \mu \mathrm{l} / \mathrm{min}$ for $5 \mathrm{~min}$.

\subsection{Neurological score}

After reperfusion for $24 \mathrm{~h}$, neurological score was measured by an observer who was unaware of the group allocation according to the Zea Longa five-point scale [33]: 0, no neurological deficit; 1 , failed to fully extend their left forepaw; 2 , circling to the left; 3 , falling to the left; 4 , failure to walk spontaneously or stroke-related death. Only rats with scores 1, 2, and 3 were used in the following experiments.

\subsection{2, 3, 5-Triphenyl-2H-tetrazolium chloride (TTC) staining}

Rats were anesthetized with $10 \%$ chloral hydrate $(300 \mathrm{mg} / \mathrm{kg})$, and brains were collected and putted on ice for 30 min after reperfusion. Next, the frozen brain tissue was sliced into $2-\mathrm{mm}$ coronal sections and stained with $1 \%$ TTC (Sigma, St. Louis, MO, USA) at $37^{\circ} \mathrm{C}$ for $15-20 \mathrm{~min}$ in the dark. The TTC-stained brain was then fixed with $4 \%$ formaldehyde for $24 \mathrm{~h}$ at room temperature and photographed with a digital camera. The infarct volume was analyzed with ImageJ 2 software (Media Cybernetics, Bethesda, MD, USA) and followed the double-blind principle.

\subsection{Slice preparation}

After reperfusion, the thoracic cavity of the anesthetized rat was first opened, and $4 \%$ paraformaldehyde was used for perfusion from the apex of the heart. Next, the perfused brain was fixed in $4 \%$ paraformaldehyde at $4^{\circ} \mathrm{C}$ for 2 days, and then immersed in a $30 \%$ sucrose solution. Finally, $30 \mu \mathrm{m}$ slices are cut out with a microtome (Thermo Scientific, Inc., New York, NY, USA).

\subsection{Immunofluorescent staining}

The brain slices were laid flat on an adhesive glass slide. Then the slices were permeabilized with $0.3 \%$ Triton X-100 for $30 \mathrm{~min}$, and blocked with goat serum for $1 \mathrm{~h}$. For immunofluorescence of cells, after the cell slices were fixed with $4 \%$ paraformaldehyde for 15 minutes, the cell slices were treated with $0.3 \%$ Triton X-100 for 5 min and $5 \%$ BSA for $1 \mathrm{~h}$. Brain slices and cell slices were incubated at $4^{\circ} \mathrm{C}$ overnight with following primary antibodies: mouse anti-GFAP (1:300; Cell Signaling Technology, Danvers, MA, USA), and rabbit anti-p65 (1:400; Cell Signaling Technology, Danvers, MA, USA). The secondary antibodies were as follows: Cy3 anti-rabbit IgG (1:100; Boster Biological Technology, Wuhan, China), FITC anti-mouse IgG (1:50; Boster Biological Technology, Wuhan, China). The immunofluorescence pictures were collected with SP8 microscope (Leica Microsystems, Germany), and the immunofluorescence density was analyzed with ImageJ software.

\subsection{Cell culture}

The human Glioma cell line U251 was provided by the Cell Resource Center Chinese Academy of Sciences (Shanghai, China) and cultured in Dulbecco's modified Eagle's Medium (DMEM) (Hyclone, China) with $10 \%$ fetal bovine serum (FBS) (Gibco, Invitrogen, USA) and $100 \mathrm{U} / \mathrm{ml}$ penicillin/streptomycin under a humidified atmosphere containing $5 \% \mathrm{CO}_{2}$ at $37^{\circ} \mathrm{C}$. 


\subsection{Oxygen-glucose deprivation/reperfusion (OGD/R) and Orexin-A (OXA) treatment}

For the OGD/R, the medium is replaced with glucose-free DMEM medium when U251 cells grow to a density of $70 \%-80 \%$, and the cells are transferred to a tri-gas incubator (Thermo Fisher Scientific) with 95 $\% \mathrm{~N}_{2}$ and $5 \% \mathrm{CO}_{2}$. After $4 \mathrm{~h}$ of OGD treatment, the cells were followed by quick reintroduction of oxygen and glucose. In addition, cells were treated with OXA $\left(1 \times 10^{-7} \mathrm{M}\right)$ for $4 \mathrm{~h}$ at the onset of reperfusion.

Cells were also treated with the inhibitors of the OX1R, SB-334867 free base (1.5 $\mu \mathrm{M}$; MedChemExpress, Monmouth Junction, NJ, USA), 30 minutes before OXA treatment. A flow chart of the in vitro study design is shown in Fig. 1.

\subsection{Hoechst 33342 staining}

After OGD/R and OXA treatment, U251 cells grown in a 12-well plate were incubated with $1 \mathrm{~mL}$ Hoechst 33342 (Solarbio, Beijing, China) at $4^{\circ} \mathrm{C}$ for $30 \mathrm{~min}$ in the dark. The picture was taken and collected using an inverted fluorescence microscope (Olympus IX 71) and ImageJ software was used to calculate the apoptosis rate.

\subsection{Cytokine Enzyme-Linked Immunosorbent Assay (ELISA)}

Culture supernatants were collected and saved at $-80^{\circ} \mathrm{C}$ until used. ELISA for IL-1 $\beta$, IL-6, TNF- $\alpha$, and IL-18 were used to measure the anti-inflammation effect of OXA.

All operations were according to the manufacturer's instructions (Beijing 4A Biotech Co., Ltd, Beijing, China).

\subsection{Western blot assay}

The protein of U251 cells and brain cortex tissue on the ischemic side were lysed and extracted using lysis buffers containing protease and phosphatase inhibitors (Cell Signaling Technology, Danvers, MA, USA). Nuclear and cytoplasmic proteins were extracted using nuclear and cytoplasmic extraction kits (Beyotime Biotechnology, Shanghai, China). Proteins were separated by 10-12\% SDS-PAGE and transferred to PVDF membranes. After blocking with $5 \%$ non-fat dry milk in TBST at room temperature for $1.5 \mathrm{~h}$, the membranes were incubated overnight at $4^{\circ} \mathrm{C}$ with the following primary antibodies: anti-pERK, anti-T-ERK, anti-p-P38, anti-P38, anti-p65, anti-iNOS, anti-TBP, anti-Bax, anti-cleaved caspase-9, anticleaved caspase-3, anti-cytc (1:1,000; Cell Signaling Technology, Danvers, MA, USA), anti-OX1R, anti-IL-1 $\beta$ (1:1000; Abcam, Cambridge, MA, USA), anti-IL-6 (1:1000; Novusbio, Colorado, USA), anti-TNF-a (1:500; Novusbio, Colorado, USA), anti-Bcl-2(1:1000; Wanleibio Co., Ltd., Shenyang, China), and anti- $\beta$-actin 
(1:1,000; Zhongshan Golden Bridge Biotechnology, Beijing, China). Then, the membranes were incubated with goat anti-rabbit secondary antibodies or goat anti-mouse secondary antibodies (1:5,000, Zhongshan Golden Bridge Biotechnology, Beijing, China) at room temperature for $1 \mathrm{~h}$. The bands were visualized using enhanced chemiluminescence system (ECL, Liankebio Biotechnology, Hangzhou, China). The grayscale intensities of protein bands were measured using the ImageJ software.

\subsection{Statistical analysis}

GraphPad Prism 5.0 (GraphPad Software, Inc., La Jolla, CA, USA) was used to analyze data, and data are expressed as means \pm SEM. One-way ANOVA followed by Tukey's test was performed. Non-normally distributed data (the neurological scores) were analyzed using the non-parametric Mann-Whitney $U$ test. All data are representative of at least three independent experiments. $p<0.05$ was considered statistically significant.

\section{Results}

\subsection{OXA reduces cerebral infarction volume and improves neurological deficit score.}

To evaluate the neuroprotective effects of OXA, neurological deficit score and 2,3,5-Triphenyltetrazoliumchloride (TTC) staining were performed on rats. As shown in Fig. 2A, TTC staining showed that neither the sham group nor the OXA group had cerebral infarction (normal cerebral tissues were stained red and infarcted areas were stained white), while the MCAO/R group had obvious cerebral infarction (34 $\pm 12 \%)$. However, infarction volume was significantly reduced by the intracerebroventricular administration of OXA (the infarct volume in MCAO/R + OXA group was $14.34 \pm 0.22 \%, p<0.001$ ).

As shown in Fig. 2B, the average neurological deficit score of the sham group and the OXA group was 0 , indicating no neurological deficit, however, the MCAO/R group increased significantly $(p<0.001)$. OXA could significantly decrease the neurological deficit score in MCAO/R+OXA group $(p<0.01)$. Therefore, OXA showed a definite neuroprotective effect on infarct volume and neurological deficit score in cerebral I/R injury.

\subsection{OGD/R increased the expression of inflammation, apoptosis-related proteins and OX1R in U251 astrocytoma cells.}

To investigate the changes in the expression of inflammation-related proteins and apoptosis-related proteins in astrocytes after cerebral I/R, we established OGD/R model in U251 astroglioma cells in vitro. Western blot was used to detect the target protein levels at different reperfusion points after $4 \mathrm{~h}$ of oxygen glucose deprivation. As shown in Fig. 3A, The expression levels of p-ERK and p-P38 sharply 
increased after OGD/R, peaking at $4 \mathrm{~h}$ and $2 \mathrm{~h}$, respectively, and maintained at high levels at $2-8 \mathrm{~h}(p<$ $0.05, p<0.01, p<0.001)$, and then gradually decreased to the base level at $12 \mathrm{~h}$. NF-kB p65, the central regulator of inflammatory response [34], its expression level significantly increased at OGD/R $2 \mathrm{~h}(p<$ $0.05)$, and reached a peak at OGD/R $4 \mathrm{~h}(p<0.01)$. INOS is a pro-inflammatory target gene of NF-KB p65 [35], and its expression level only increased significantly at OGD/R $4 \mathrm{~h}(p<0.001)$, which corresponds to the highest expression level of NF-KB p65 at OGD/R $4 \mathrm{~h}$. These results indicate that the MAPK signaling pathway and the NF-KB p65 signaling pathway were involved in the OGD/R process, and both maintain high activity at OGD/R $4 \mathrm{~h}$.

As shown in Fig. 3B, the ratio of Bcl-2/Bax was significantly lower at $2 \mathrm{~h}, 4 \mathrm{~h}, 6 \mathrm{~h}$ and $8 \mathrm{~h}$ in OGD/R model compared with the control group $(p<0.01, p<0.001)$, which indicated that the balance between antiapoptosis and pro-apoptosis was broken and turned to apoptosis in the U251 astroglioma cells treated with OGD/R.

OX1R, the receptor for the neuropeptide OXA, is low expressed in U251 astroglioma cells without any treatment. Interestingly, the expression of OX1R was significantly enhanced at OGD/R $2 \mathrm{~h}, 4 \mathrm{~h}, 6 \mathrm{~h}$, and 8 $\mathrm{h}(p<0.01, p<0.001$; Fig. $3 \mathrm{C})$, which is consistent with the above-mentioned changes in the expression levels of $\mathrm{p}$-ERK, $\mathrm{p}$-P38 and Bcl-2/Bax. Therefore, these results indicate that OXA may reduce cerebral I/R injury by regulating the inflammatory response mediated by MAPK and NF-KB p65 signaling pathways in conjunction with OX1R, and OGD/R $4 \mathrm{~h}$ was chosen to verify this hypothesis in the following experiments.

\subsection{OXA inhibited the production of pro-inflammatory factors in OGD/R U251 astrocytoma cells and MCAO/R rats.}

To verify the anti-inflammatory effects of OXA, we collected the cultured supernatants from OGD/Rtreated U251 astroglioma cells treated with OXA or not for ELISA analysis to detect the levels of proinflammatory cytokines. Compared with the control group, the concentration of IL-1 $\beta$, TNF-a, IL- 6 and IL18 released into the supernatant in the OGD/R group sharply increased $(p<0.01, p<0.001$; Fig. $4 \mathrm{~A})$, and the concentration of IL- 6 increased by 78.95 times. However, the levels of these proinflammatory cytokines were dramatically decreased in the OGD/R+ OXA group $(p<0.05, p<0.01, p<0.001$; Fig. 4A). Then western blot was executed to evaluate the expression levels of IL-1 $\beta$, TNF- $a$, IL- 6 and iNOS in vivo and in vitro. The levels of IL-1 $\beta$, TNF-a, IL- 6 and iNOS were increased after OGD/R $(p<0.05, p<0.001$; Fig. 4B), but we found that $O X A$ significantly reversed the increased pro-inflammatory factors expression $(p<0.05, p<0.01$; Fig. 4B). Similarly, in the MCAO/R rat, the levels of IL-1 $\beta$, TNF-a, IL-6 and iNOS were significantly decreased with OXA treatment compared with MCAO/R group $(p<0.05, p<0.01$; Fig. $4 C)$. These results indicate that OXA decreased the production and release of proinflammatory factors in vivo and in vitro.

\subsection{OXA inhibited the excessive activation of astrocytes in MCAO/R rats.}


Glial fibrillary acidic protein (GFAP) is the hallmark intermediate filament protein in astrocytes and its upregulation is characteristic of astrocyte activation [36]. Both immunofluorescence and WB results showed that the expression level of GFAP increased significantly in the ischemic cerebral cortex of rats $(p<0.01$, $p<0.001$; Fig. 5), which indirectly indicated that cerebral I/R injury caused the activation and proliferation of astrocytes. However, OXA down-regulated the expression of $\operatorname{GFAP}(p<0.05 ;$ Fig. 5$)$ and relieved excessive activation of astrocytes.

\subsection{OXA inhibited the nuclear translocation of NF-kB p65 in OGD/R U251 astrocytoma cells and MCAO/R rats.}

To explore and verify the cellular mechanism of OXA anti-inflammation, immunofluorescence staining and western blot were performed to clarify the potential effect of OXA on the NF-KB p65 in the MCAO/R rat and U251 astroglioma cells subjected to OGD/R. NF-KB signaling is a key pathway in the inflammatory response [14], and NF-KB p65 is transferred from the cytoplasm to the nucleus to regulate the transcription of inflammatory factors when NF-KB signaling is activated $[18,19]$. As shown in Fig. 6A, immunofluorescence staining of p65 and GFAP showed that OXA remarkably decreased the nuclear translocation of $\mathrm{p} 65$ that was induced by OGD/R in U251 astroglioma cells $(p<0.05$; Fig. $6 \mathrm{~A})$. Further, we detected the expression of NF-KB p65 in cytosolic and nuclear extracts by Western blot. The results showed that the nuclear translocation levels of p65 were significantly declined in OXA treated groups $(p<$ 0.001 ; Fig. 6B). This result was also confirmed in MCAO/R + OXA group $(p<0.001$; Fig. 6C). These observations demonstrated that OXA inhibited the nuclear translocation of NF-KB p65 and downregulated NF-kB signaling in cerebral I/R injury.

\subsection{OXA inhibited MAPK signaling pathway in OGD/R U251 astrocytoma cells and MCAO/R rats.}

p-ERK and p-p38 play crucial roles in the MAPK signaling pathway by sending inflammatory signals [15]. So, western blot was used to confirm the effect of OXA on the MAPK pathway. As shown in Fig. 7A, the levels of $p$-ERK/ERK and p-p38/p38 were significantly increased in OGD/R group $(p<0.001$; Fig. 7A), while OXA markedly suppressed these OGD/R-induced changes $(p<0.01 ; \mathrm{Fig}$. 7A). Moreover, in the $\mathrm{MCAO} / \mathrm{R}$ rat, treatment with OXA markedly down-regulated the levels of $p$-ERK/ERK and p-p38/p38 compared with those treated with vehicle ( $p<0.01$; Fig. 7B). These results indicate that OXA downregulated the $\mathrm{p}$-ERK and p-p38 signaling in the MAPK signaling pathway.

\subsection{OX1R mediated the effect of OXA on inhibiting MAPK and NF-KB p65 signaling pathway.}

Next, we proceed to verify whether OXA down-regulates the MAPK and NF-KB p65 signaling pathways are dependent on OX1R, for which we added antagonist of OX1R SB-334867 and OXA co-treatment groups. Western blot was used to detect changes in the expression of MAPK and the nuclear translocation of NFKB p65 after SB-334867 intervention. As shown in Fig. 8A, the expression of p-ERK and p-p38 in the 
$\mathrm{OGD} / \mathrm{R}+\mathrm{OXA}+\mathrm{SB}$ group increased compared with the OGD/R + OXA group $(p<0.05, p<0.001$; Fig. 8A). Moreover, the intervention of the antagonist SB-334867 increased the nuclear translocation of NF-KB p65 than in the OGD/R+ OXA group $(p<0.05$; Fig. 8B). It is indicated that OX1R mediated the regulation of OXA on MAPK and $p 65$ signaling pathways.

\subsection{OXA inhibited apoptosis in OGD/R U251 astrocytoma cells and MCAO/R rats.}

To observe the effect of anti-apoptosis of OXA in cerebral I/R injury, apoptosis-related proteins were detected by western blot, and the apoptosis rate was measured by Hoechst 33342 staining in U251 astrocytoma cells. The Hoechst 33342 staining show that the percentage of apoptotic cells was significantly increased in U251 astrocytoma cells after OGD/R ( $p<0.001$; Fig. 9A), and OXA effectively inhibited this process $(p<0.01$; Fig. 9A), showing an obvious anti-apoptosis effect.

Western blot analysis revealed that the Bcl-2/Bax ratio was significantly reduced in the OGD/R group compared with the control group ( $p<0.001$; Fig. 9B). However, treatment with OXA significantly enhanced the $\mathrm{Bcl}-2 / \mathrm{Bax}$ ratio compared with the OGD/R group ( $p<0.05$; Fig. 9B). Furthermore, the same change trend of $\mathrm{Bcl}-2 / \mathrm{Bax}$ was observed in $\mathrm{MCAO} / \mathrm{R}$ rats. The $\mathrm{Bcl}-2 / \mathrm{Bax}$ ratio was also significantly increased following treatment with OXA compared with the I/R group ( $p<0.05$; Fig. $9 \mathrm{C})$.

12 Western blot analysis revealed that the expression of cytochrome $C$, cleaved caspase- 9 and cleaved caspase-3 in the OGD/R group was significantly higher than in the control group $(p<0.05, p<0.001$; Fig. 9B). Treatment with OXA reversed the effect of OGD/R on cytochrome $C$, cleaved caspase-9 and cleaved caspase-3 induction ( $p<0.05, p<0.001$; Fig. 9B). Similarly, the intracerebroventricular administration of OXA was able to reverse the effect of MCAO/R on cytochrome $C$, cleaved caspase- 9 and cleaved caspase 3 induction ( $p<0.05, p<0.01$ and $p<0.001$; Fig. 9 C). Therefore, the above results indicate that OXA possessed anti-apoptotic effect in cerebral I/R injury.

\section{Discussion}

The present study showed that OXA protected the brain against I/R injury by reducing cerebral infarction volume and improving neurological function score, which is consistent with previous reports [27, 28]. However, the highlight is that we found that OXA exerted anti-inflammatory effects by reducing the expression of inflammatory factors, and inhibited the excessive activation of astrocytes in the cortex of rats after cerebral I/R injury. As an important neuroprotective agent, OXA has a significant effect of antiapoptosis consistent with our prior research [31]. Here, we found that OXA could effectively reduce apoptosis expression and inflammation caused by OGD/R in U251 astrocytoma cells. To the best of our knowledge, this is the first research focusing on the mechanisms of the effects of OXA on astrocytes.

Increasing evidences have suggests that the inflammatory response leads to secondary brain damage following cerebral I/R [37]. Immune effector cells from the cerebral cortex will produce and release a large number of pro-inflammatory factors, such as IL-1 $\beta$ and TNF-a. These pro-inflammatory factors will 
initiate and aggravate the inflammatory response and further aggravate brain damage [38]. Therefore, reducing the production and release of pro-inflammatory factors is an effective means to alleviate cerebral I/R injury. Inflammation is also considered to be one of the main targets for the development of new stroke treatments [39]. Previous reports have shown that treatment with exogenous IL-1 $\beta$ can aggravate brain damage [40]. TNF- $a$ is one of the most widely studied cytokines. Administration of TNF-a during ischemic brain injury could exacerbate the damage, and administration of TNF-neutralizing antibodies can reduce this damage [41]. The up-regulation of iNOS expression can lead to excessive production of NO after cerebral ischemia, and the massive production of NO causes irreversible damage to cells by inhibiting the mitochondrial respiratory chain and forming peroxynitrite with superoxide anions [42-44]. Moreover, a growing amount of evidence demonstrates that neurotoxicity caused by ischemia could be alleviated by iNOS inhibitors $[45,46]$. In this study we found that OXA treatment significantly decreased expression levels of TNF- $\alpha$, IL-1 $\beta, I L-6$ and iNOS induced by MCAO/R. These results show that OXA has neuroprotective effects against I/R injury by preventing pro-inflammatory cytokines.

Astrocytes are the most ubiquitous type of nerve cells in the brain, which are implicated in pathological process of ischemic stroke. Cerebral ischemia activates astrocytes. However, reactive astrocytes are considered to be detrimental to the neurological outcome after stroke [9]. The activated astrocytes release many pro-inflammatory factors, and then a large number of pro-inflammatory factors trigger and amplify the inflammatory response, and further activate astrocytes to form a cycle [12]. It is generally believed that reactive astrocytes in the early stage of central nervous system damage are beneficial to neurons. They support neurons by participating in a variety of biological processes such as regulating inflammation and neurotransmitter nutrition. However, over-activated astrocytes can accelerate the process of neuronal death and infarction $[9,47]$. Astrocyte activation is manifested by astrocyte proliferation, morphological changes and enhanced expression of GFAP. In this study, we observed the proliferation of astrocytes in the infarcted cortex, and the expression of astrocyte marker GFAP also increased significantly, which was abolished by the OXA treatment. This indicates that the activation of astrocytes plays an important role in cerebral I/R injury, and OXA inhibits the excessive activation of astrocytes to exert neuroprotective effects. Accumulating evidence strongly suggests that astrocytemediated inflammation can cause secondary damage to the brain after stroke [12, 13]. Therefore, inhibiting the inflammatory response mediated by astrocytes can treat neuroinflammation after stroke. Moreover, recent studies have shown that inhibiting astrocyte-mediated inflammation can reduce cerebral $I / R$ injury $[48,49]$. Consistent with the results of these studies, the level of pro-inflammatory factors was up-regulated in our study when induced by OGD/R and MCAO/R, but their expressed were showed at lower levels after OXA treatment. These results indicate that OXA inhibits neuroinflammation by downregulating the levels of pro-inflammatory factors, which is mediated by astrocytes.

Increasing evidences have reported that NF-KB signaling pathway is implicated in ischemic stroke [16, 50, 51]. As a pivot regulator of inflammation, NF-KB plays a key role in the inflammatory cascade [16]. It regulates the transcription of downstream target genes, including TNF- $a$, IL-1 $1 \beta$, IL- 6 and iNOS, which promotes the expression and release of pro-inflammatory factors $[18,19]$, finally contributing to neuronal death, and exacerbating cerebral I/R injury $[52,53]$. Therefore, the downregulation of NF-kB signaling 
pathway alleviates brain damage induced by I/R $[16,54]$. The nuclear translocation of NF-KB subunit p65 is one of the indicators that can directly and accurately show that the NF-KB signaling pathway is activated [55]. In this experiment, through the p65 and GFAP immunofluorescence images with U251 astroglioma cells subjected to $\mathrm{OGD} / \mathrm{R}$, we can clearly observe that the red fluorescence of p 65 was significantly increased in the nucleus, while the fluorescence intensity of the cytoplasm was greatly reduced. The nucleoplasmic protein extraction and western blot analysis in U251 astroglioma cells after $\mathrm{OGD} / \mathrm{R}$ and rat ischemic cortex were also consistent with it. These findings can fully explain that p65 was activated. However, treatment with OXA decreased nuclear translocation of p65 in U251 astrocytoma cells following $\mathrm{OGD} / \mathrm{R}$, indicating that OXA exerts anti-inflammatory effects by inhibiting NF-KB. In vivo experiments in MCAO/R rats also supported this, but it must be emphasized that the anti-inflammatory effect of OXA in vivo not only have affected astrocytes, but may have include microglia and neurons. Further work is necessary to illustrate this point. The anti-inflammatory effect of OXA can increase neuronal tolerance to ischemia and reduce neuronal damage in cerebral I/R injury.

MAPK signaling pathway is involved in inflammatory processes of cerebral $\mathrm{I} / \mathrm{R}$ injury [56], and is the main signaling mechanism that regulates neuroinflammation [57]. The activated MAPK causes the overproduction of pro-inflammatory factors [58]. In contrast with ERK, which is responsible for cell survival and proliferation, the p38 is more closely related to the production of pro-inflammatory factors [59]. In our study, the phosphorylation of ERK and p38 obviously increased at different reperfusion times in U251 astrocytoma cells after OGD/R. This indicates that the p-ERK and p38 signaling pathways are activated, and it can be inferred that the MAPK pathway in astrocytes is also involved in cerebral I/R injury. A similar phenomenon was observed in the MCAO/R rat. However, OXA inhibited the activation of $\mathrm{p}$-ERK and p-p38. So, we have reason to infer that OXA reduces the production and release of proinflammatory factors by down-regulating the p-ERK and p-p38 signaling pathways.

To a certain extent, SB334867, an antagonist of OX1R, abolished the effect of OXA down-regulating the ERK and p38 pathways and inhibiting p65 nuclear translocation in OGD/R U251 astrocytoma cells. Our findings indicate that this anti-inflammatory effect of OXA is dependent on OX1R. However, it also needs to be verified in vivo. Therefore, there is an additional strong experimental evidence supporting OXA to function through OX1R [25].

Recent reports indicate that astrocyte apoptosis is involved in the pathological process of cerebral ischemia [8]. In fact, astrocytes were the first to suffer an ischemic blow because their end feet directly surround the capillaries. Astrocytes play an important role in neuroprotection and survival of neurons, and its dysfunction seriously affects the survival ability of neurons. There is considerable evidence that neuron and astrocyte apoptosis coexist in cerebral ischemia and neurodegenerative diseases [7]. Therefore, the study of regulating astrocyte apoptosis is particularly important. The excessive production of ROS in ischemic stroke causes the release of cytochrome $\mathrm{C}$ from the mitochondrial membrane into the cytoplasm [60], which activate caspase-9 and consequently activate caspase-3, DNA-breaking enzymes and repair enzymes, leading to cell death [61-63]. The opening of mitochondrial permeability transition pores (PTP) is the key to the release of cytochrome $\mathrm{C}$ into the cytoplasm. PTP, which are channels across 
the inner and outer mitochondrial membranes, are also channels for the release of pro-apoptotic proteins including cytochrome $\mathrm{C}$ [64]. Various factors, such as $\mathrm{Ca}^{2+}$, reactive oxygen species, adenine nucleotides, inorganic phosphate, etc., can induce the formation of PTP, which is enhanced by Bax and inhibited by $\mathrm{Bcl}-2$ [65]. Studies have reported that the ratio of $\mathrm{Bcl}-2 / \mathrm{Bax}$ is reduced in the rat brain following cerebral I/R $[66,67]$. In the cortical tissue of MCAO/R rats, the expression level of Bcl-2 was significantly decreased and the level of Bax was significantly increased, so that the ratio of $\mathrm{Bcl}-2 / \mathrm{Bax}$ decreased, which is consistent with the results of previous studies. Similarly, the ratio of $\mathrm{Bcl}-2 / \mathrm{Bax}$ was also reduced in OGD/R U251 glioma cells. However, after OXA treatment, the expression level of Bcl-2 increased while the expression level of Bax decreased, and the ratio of Bcl-2/Bax increased accordingly. Moreover, OXA inhibited the expression of cytochrome $C$, cleaved caspase- 9 and cleaved caspase- 3 induced by OGD/R in U251 glioma cells and MCAO/R in rat. In addition, Hoechst 33342 staining showed that OXA did reduced the rate of apoptosis caused by OGD/R. Therefore, from the above results, we infer that OXA alleviates astrocyte apoptosis by regulating the ratio of $\mathrm{Bcl}-2 / \mathrm{Bax}$ and inhibiting the expression of cytochrome $\mathrm{C}$, cleaved caspase- 9 and cleaved caspase-3. It still needs to be pointed out that the anti-apoptotic effects of OXA in vivo are multifaceted, which may include neurons, astrocytes, microglia and other nerve cells.

\section{Conclusions}

In conclusion, OXA has a neuroprotective effect against cerebral I/R injury. As shown in Fig. 10, our findings indicate that OXA attenuated astrocytes apoptosis, astrocytes activation and pro-inflammatory cytokines production after OGD/R and I/R injury. This effect depends on OX1R and may be due to the inhibition of nuclear translocation of NF-KB and the inhibition of phosphorylation of MAPK/ERK and MAPK/p38. Our results enrich the molecular mechanisms of OXA underlying the neuroprotective effect on cerebral I/R injury, which will provide promising drug targets for the treatment of stroke.

\section{Abbreviations}

OXA: Orexin-A; CIRI: Cerebral ischemia/reperfusion injury; MCAO/R: Middle cerebral artery occlusion and reperfusion; OGD/R: Oxygen glucose deprivation and reperfusion; TTC: 2,3,5-triphenyltetrazolium chloride; NF-kB: Nuclear factor-Kb; MAPK: Mitogen-activated protein kinase; AIS: Acute ischemic stroke; rtPA: Recombinant tissue plasminogen activator; I/R: Ischemia/reperfusion; TNF-a: Tumor necrosis factor-a; ROS: Reactive oxygen species; iNOS: Inducible nitric oxide synthase; JNKs: c-Jun N-terminal kinases; ERK: Extracellular signal-regulated kinase; OXB: Orexin-B; GPCRs: G-protein-coupled receptors; OX1R: Orexin receptor type 1; OX2R: Orexin receptor type 2; CNS: Central nervous system; SD: Sprague-Dawley; MCAO: Middle cerebral artery occlusion; ECA: External carotid artery; ICA: Internal carotid artery; ICV: Intracerebroventricular; DMEM: Dulbecco's modified Eagle's Medium; FBS: Fetal bovine serum; ELISA: Cytokine Enzyme-Linked Immunosorbent Assay; ECL: Enhanced chemiluminescence system; GFAP: Glial fibrillary acidic protein; SB: SB-334867; PTP: Permeability transition pores.

\section{Declarations}




\section{Ethics approval and consent to participate}

All animal experiments were conducted in accordance with the National Institutes of Health Guide for the Care and Use of Laboratory Animals, and approved by the Animal Ethics Committee of Jining Medical University, China (approval no.2018-JS-001).

\section{Consent for publication}

Not applicable.

\section{Acknowledgements}

Not applicable.

\section{Availability of data and materials}

Not applicable.

\section{Authors' contribution}

Chunmei Wang conceived and designed the experiments; Dandan Xu and Tingting Kong conducted the experiments and wrote the manuscript; Ziqi Shao and Rumin Zhang assisted in the preparation of animal models; Shengnan Zhang and Baohua Cheng assisted in the analysis of the data; Qingxia Kong and Jing Chen revised the manuscript.

\section{Funding}

This work was supported by Research Fund for Academician Lin He New Medicine (JYHL2019ZD02) and the Natural Science Foundation of Shandong Province (ZR2020MH136, ZR2020QC079, ZR2020QC080).

\section{Competing interests}

The author(s) declared no potential conflicts of interest with respect to the research, authorship, and/or publication of this article.

\section{References}

1. P. Khandelwal, D.R. Yavagal, R.L. Sacco, Acute Ischemic Stroke Intervention, J Am Coll Cardiol 67(22) (2016) 2631-44.

2. A.A. Rabinstein, Treatment of Acute Ischemic Stroke, Continuum (Minneap Minn) 23(1, Cerebrovascular Disease) (2017) 62-81.

3. M. Nour, F. Scalzo, D.S. Liebeskind, Ischemia-reperfusion injury in stroke, Interv Neurol 1(3-4) (2013) 185-99. 
4. J.E. Jung, G.S. Kim, H. Chen, C.M. Maier, P. Narasimhan, Y.S. Song, K. Niizuma, M. Katsu, N. Okami, H. Yoshioka, H. Sakata, C.E. Goeders, P.H. Chan, Reperfusion and neurovascular dysfunction in stroke: from basic mechanisms to potential strategies for neuroprotection, Mol Neurobiol 41(2-3) (2010) $172-9$.

5. K.L. Lambertsen, K. Biber, B. Finsen, Inflammatory cytokines in experimental and human stroke, Journal of cerebral blood flow and metabolism : official journal of the International Society of Cerebral Blood Flow and Metabolism 32(9) (2012) 1677-98.

6. D.B. Tower, O.M. Young, The activities of butyrylcholinesterase and carbonic anhydrase, the rate of anaerobic glycolysis, and the question of a constant density of glial cells in cerebral cortices of various mammalian species from mouse to whale, J Neurochem 20(2) (1973) 269-78.

7. K. Takuma, A. Baba, T. Matsuda, Astrocyte apoptosis: implications for neuroprotection, Prog Neurobiol 72(2) (2004) 111-27.

8. R.G. Giffard, R.A. Swanson, Ischemia-induced programmed cell death in astrocytes, Glia 50(4) (2005) 299-306.

9. Z. Liu, M. Chopp, Astrocytes, therapeutic targets for neuroprotection and neurorestoration in ischemic stroke, Prog Neurobiol 144 (2016) 103-20.

10. C.P. Wang, Y.W. Shi, M. Tang, X.C. Zhang, Y. Gu, X.M. Liang, Z.W. Wang, F. Ding, Isoquercetin Ameliorates Cerebral Impairment in Focal Ischemia Through Anti-Oxidative, Anti-Inflammatory, and Anti-Apoptotic Effects in Primary Culture of Rat Hippocampal Neurons and Hippocampal CA1 Region of Rats, Mol Neurobiol 54(3) (2017) 2126-2142.

11. S. Hostenbach, M. Cambron, M. D'Haeseleer, R. Kooijman, J. De Keyser, Astrocyte loss and astrogliosis in neuroinflammatory disorders, Neurosci Lett 565 (2014) 39-41.

12. J. Qiu, Z. Yan, K. Tao, Y. Li, Y. Li, J. Li, Y. Dong, D. Feng, H. Chen, Sinomenine activates astrocytic dopamine D2 receptors and alleviates neuroinflammatory injury via the CRYAB/STAT3 pathway after ischemic stroke in mice, Journal of neuroinflammation 13(1) (2016) 263.

13. M. Pekny, M. Pekna, A. Messing, C. Steinhauser, J.M. Lee, V. Parpura, E.M. Hol, M.V. Sofroniew, A. Verkhratsky, Astrocytes: a central element in neurological diseases, Acta Neuropathol 131(3) (2016) 323-45.

14. J.J. Cho, Z. Xu, U. Parthasarathy, T.T. Drashansky, E.Y. Helm, A.N. Zuniga, K.J. Lorentsen, S. Mansouri, J.Y. Cho, M.J. Edelmann, D.M. Duong, T. Gehring, T. Seeholzer, D. Krappmann, M.N. Uddin, D. Califano, R.L. Wang, L. Jin, H. Li, D. Lv, D. Zhou, L. Zhou, D. Avram, Hectd3 promotes pathogenic Th17 lineage through Stat3 activation and Malt1 signaling in neuroinflammation, Nat Commun 10(1) (2019) 701.

15. B.H. Clausen, K.L. Lambertsen, F. Dagnaes-Hansen, A.A. Babcock, C.U. von Linstow, M. Meldgaard, B.W. Kristensen, T. Deierborg, B. Finsen, Cell therapy centered on IL-1Ra is neuroprotective in experimental stroke, Acta Neuropathol 131(5) (2016) 775-91.

16. D.A. Ridder, M. Schwaninger, NF-kappaB signaling in cerebral ischemia, Neuroscience 158(3) (2009) 995-1006. 
17. Y. Huang, W. Mei, J. Chen, T. Jiang, Z. Zhou, G. Yin, J. Fan, Gamma-secretase inhibitor suppressed Notch1 intracellular domain combination with p65 and resulted in the inhibition of the NF-kappaB signaling pathway induced by IL-1beta and TNF-alpha in nucleus pulposus cells, J Cell Biochem (2018).

18. M. Das, J. Lu, M. Joseph, R. Aggarwal, S. Kanji, B.K. McMichael, B.S. Lee, S. Agarwal, A. RayChaudhury, O.H. Iwenofu, P. Kuppusamy, V.J. Pompili, M.K. Jain, H. Das, Kruppel-like factor 2 (KLF2) regulates monocyte differentiation and functions in $\mathrm{mBSA}$ and IL-1 beta-induced arthritis, Curr Mol Med 12(2) (2012) 113-25.

19. S. Liu, X. Wang, L. Pan, W. Wu, D. Yang, M. Qin, W. Jia, C. Xiao, F. Long, J. Ge, X. Liu, Y. Zhu, Endogenous hydrogen sulfide regulates histone demethylase JMJD3-mediated inflammatory response in LPS-stimulated macrophages and in a mouse model of LPS-induced septic shock, Biochem Pharmacol 149 (2018) 153-162.

20. Y. Sun, W.Z. Liu, T. Liu, X. Feng, N. Yang, H.F. Zhou, Signaling pathway of MAPK/ERK in cell proliferation, differentiation, migration, senescence and apoptosis, J Recept Signal Transduct Res 35(6) (2015) 600-4.

21. E.K. Kim, E.J. Choi, Compromised MAPK signaling in human diseases: an update, Arch Toxicol 89(6) (2015) 867-82.

22. T. Sakurai, A. Amemiya, M. Ishii, I. Matsuzaki, R.M. Chemelli, H. Tanaka, S.C. Williams, J.A. Richardson, G.P. Kozlowski, S. Wilson, J.R.S. Arch, R.E. Buckingham, A.C. Haynes, S.A. Carr, R.S. Annan, D.E. McNulty, W.-S. Liu, J.A. Terrett, N.A. Elshourbagy, D.J. Bergsma, M. Yanagisawa, Addendum, Cell 92(5) (1998).

23. K. Ohno, M. Hondo, T. Sakurai, Cholinergic regulation of orexin/hypocretin neurons through $M(3)$ muscarinic receptor in mice, J Pharmacol Sci 106(3) (2008) 485-91.

24. T. Sakurai, The neural circuit of orexin (hypocretin): maintaining sleep and wakefulness, Nat Rev Neurosci 8(3) (2007) 171-81.

25. M. Laburthe, T. Voisin, A. El Firar, Orexins/hypocretins and orexin receptors in apoptosis: a minireview, Acta Physiol (Oxf) 198(3) (2010) 393-402.

26. S. Ammoun, T. Holmqvist, R. Shariatmadari, H.B. Oonk, M. Detheux, M. Parmentier, K.E. Akerman, J.P. Kukkonen, Distinct recognition of OX1 and OX2 receptors by orexin peptides, J Pharmacol Exp Ther 305(2) (2003) 507-14.

27. E. Kitamura, J. Hamada, N. Kanazawa, J. Yonekura, R. Masuda, F. Sakai, H. Mochizuki, The effect of orexin-A on the pathological mechanism in the rat focal cerebral ischemia, Neurosci Res 68(2) (2010) 154-7.

28. C.-M. Wang, Y.-Y. Pan, M.-H. Liu, B.-H. Cheng, B. Bai, J. Chen, RNA-seq expression profiling of rat MCAO model following reperfusion Orexin-A, Oncotarget 8(68) (2017) 113066-113081.

29. M. Bulbul, R. Tan, B. Gemici, G. Ongut, V.N. Izgut-Uysal, Effect of orexin-a on ischemia-reperfusioninduced gastric damage in rats, J Gastroenterol 43(3) (2008) 202-7. 
30. Y. Ogawa, Y. Irukayama-Tomobe, N. Murakoshi, M. Kiyama, Y. Ishikawa, N. Hosokawa, H. Tominaga, S. Uchida, S. Kimura, M. Kanuka, M. Morita, M. Hamada, S. Takahashi, Y. Hayashi, M. Yanagisawa, Peripherally administered orexin improves survival of mice with endotoxin shock, Elife 5 (2016).

31. T. Kong, K. Qiu, M. Liu, B. Cheng, Y. Pan, C. Yang, J. Chen, C. Wang, Orexin-A protects against oxygenglucose deprivation/reoxygenation-induced cell damage by inhibiting endoplasmic reticulum stressmediated apoptosis via the Gi and PI3K signaling pathways, Cell Signal 62 (2019) 109348.

32. L.B. Yuan, H.L. Dong, H.P. Zhang, R.N. Zhao, G. Gong, X.M. Chen, L.N. Zhang, L. Xiong, Neuroprotective effect of orexin-A is mediated by an increase of hypoxia-inducible factor- 1 activity in rat, Anesthesiology 114(2) (2011) 340-54.

33. E.Z. Longa, P.R. Weinstein, S. Carlson, R. Cummins, Reversible middle cerebral artery occlusion without craniectomy in rats, Stroke 20(1) (1989) 84-91.

34. Q. Wang, X.N. Tang, M.A. Yenari, The inflammatory response in stroke, J Neuroimmunol 184(1-2) (2007) 53-68.

35. C. Bottex-Gauthier, S. Pollet, A. Favier, D.R. Vidal, Les facteurs de transcription Rel/NF-B : rôle complexe dans les régulations cellulaires, Pathologie Biologie 50(3) (2002) 204-211.

36. E.M. Hol, M. Pekny, Glial fibrillary acidic protein (GFAP) and the astrocyte intermediate filament system in diseases of the central nervous system, Curr Opin Cell Biol 32 (2015) 121-30.

37. D. Petrovic-Djergovic, S.N. Goonewardena, D.J. Pinsky, Inflammatory Disequilibrium in Stroke, Circ Res 119(1) (2016) 142-58.

38. C. Chen, T. Li, Y. Zhao, Y. Qian, X. Li, X. Dai, D. Huang, T. Pan, L. Zhou, Platelet glycoprotein receptor Ib blockade ameliorates experimental cerebral ischemia-reperfusion injury by strengthening the bloodbrain barrier function and anti-thrombo-inflammatory property, Brain Behav Immun 69 (2018) 255263.

39. K.L. Lambertsen, B. Finsen, B.H. Clausen, Post-stroke inflammation-target or tool for therapy?, Acta Neuropathol 137(5) (2019) 693-714.

40. S.A. Loddick, N.J. Rothwell, Neuroprotective effects of human recombinant interleukin-1 receptor antagonist in focal cerebral ischaemia in the rat, Journal of cerebral blood flow and metabolism : official journal of the International Society of Cerebral Blood Flow and Metabolism 16(5) (1996) 93240.

41. M.A. Yenari, J. Liu, Z. Zheng, Z.S. Vexler, J.E. Lee, R.G. Giffard, Antiapoptotic and anti-inflammatory mechanisms of heat-shock protein protection, Ann N Y Acad Sci 1053 (2005) 74-83.

42. C. Cazevielle, A. Muller, F. Meynier, C. Bonne, Superoxide and nitric oxide cooperation in hypoxia/ reoxygenation-induced neuron injury, Free Radical Biology and Medicine 14(4) (1993) 389-395.

43. G.C. Brown, V. Borutaite, Inhibition of mitochondrial respiratory complex I by nitric oxide, peroxynitrite and S-nitrosothiols, Biochim Biophys Acta 1658(1-2) (2004) 44-9.

44. B. Sekhon, C. Sekhon, M. Khan, S.J. Patel, I. Singh, A.K. Singh, N-Acetyl cysteine protects against injury in a rat model of focal cerebral ischemia, Brain Research 971(1) (2003) 1-8. 
45. K. Mori, H. Togashi, K.-i. Ueno, M. Matsumoto, M. Yoshioka, Aminoguanidine prevented the impairment of learning behavior and hippocampal long-term potentiation following transient cerebral ischemia, Behavioural Brain Research 120(2) (2001) 159-168.

46. A. Cárdenas, J. De Alba, M.a.A. Moro, J.C. Leza, P. Lorenzo, I. Lizasoain, Protective effect of N-(3(aminomethyl)benzyl)acetamidine, an inducible nitric oxide synthase inhibitor, in brain slices exposed to oxygen-glucose deprivation, Eur J Pharmacol 354(2-3) (1998) 161-165.

47. I.P. Karve, J.M. Taylor, P.J. Crack, The contribution of astrocytes and microglia to traumatic brain injury, Br J Pharmacol 173(4) (2016) 692-702.

48. H. Liu, X. Wu, J. Luo, X. Wang, H. Guo, D. Feng, L. Zhao, H. Bai, M. Song, X. Liu, W. Guo, X. Li, L. Yue, B. Wang, Y. Qu, Pterostilbene Attenuates Astrocytic Inflammation and Neuronal Oxidative Injury After Ischemia-Reperfusion by Inhibiting NF-kappaB Phosphorylation, Front Immunol 10 (2019) 2408.

49. X. Li, L. Huang, G. Liu, W. Fan, B. Li, R. Liu, Z. Wang, Q. Fan, W. Xiao, Y. Li, W. Fang, Ginkgo diterpene lactones inhibit cerebral ischemia/reperfusion induced inflammatory response in astrocytes via TLR4/NF-kappaB pathway in rats, J Ethnopharmacol 249 (2020) 112365.

50. O.A. Harari, J.K. Liao, NF-kappaB and innate immunity in ischemic stroke, Ann N Y Acad Sci 1207 (2010) 32-40.

51. X. Hu, O. Nesic-Taylor, J. Qiu, H.C. Rea, R. Fabian, D.K. Rassin, J.R. Perez-Polo, Activation of nuclear factor-kappaB signaling pathway by interleukin-1 after hypoxia/ischemia in neonatal rat hippocampus and cortex, J Neurochem 93(1) (2005) 26-37.

52. B. Zhang, C. Song, B. Feng, W. Fan, Neuroprotection by triptolide against cerebral ischemia/reperfusion injury through the inhibition of NF-kappaB/PUMA signal in rats, Ther Clin Risk Manag 12 (2016) 817-24.

53. X. Feng, S. Yang, J. Liu, J. Huang, J. Peng, J. Lin, J. Tao, L. Chen, Electroacupuncture ameliorates cognitive impairment through inhibition of NF-kappaB-mediated neuronal cell apoptosis in cerebral ischemia-reperfusion injured rats, Molecular medicine reports 7(5) (2013) 1516-22.

54. O. Herrmann, B. Baumann, R. de Lorenzi, S. Muhammad, W. Zhang, J. Kleesiek, M. Malfertheiner, M. Kohrmann, I. Potrovita, I. Maegele, C. Beyer, J.R. Burke, M.T. Hasan, H. Bujard, T. Wirth, M. Pasparakis, M. Schwaninger, IKK mediates ischemia-induced neuronal death, Nat Med 11(12) (2005) 1322-9.

55. J.P. Pradere, C. Hernandez, C. Koppe, R.A. Friedman, T. Luedde, R.F. Schwabe, Negative regulation of NF-kappaB p65 activity by serine 536 phosphorylation, Sci Signal 9(442) (2016) ra85.

56. M. Jiang, J. Li, Q. Peng, Y. Liu, W. Liu, C. Luo, J. Peng, J. Li, K.K. Yung, Z. Mo, Neuroprotective effects of bilobalide on cerebral ischemia and reperfusion injury are associated with inhibition of proinflammatory mediator production and down-regulation of JNK1/2 and p38 MAPK activation, Journal of neuroinflammation 11 (2014) 167.

57. H.K. Kimelberg, B.A. Macvicar, H. Sontheimer, Anion channels in astrocytes: biophysics, pharmacology, and function, Glia 54(7) (2006) 747-57.

58. S.W. Himaya, B. Ryu, Z.J. Qian, Y. Li, S.K. Kim, 1-(5-bromo-2-hydroxy-4-methoxyphenyl)ethanone [SE1] suppresses pro-inflammatory responses by blocking NF-kappaB and MAPK signaling pathways in 
activated microglia, Eur J Pharmacol 670(2-3) (2011) 608-16.

59. J.B. Kim, Y.M. Yu, S.W. Kim, J.K. Lee, Anti-inflammatory mechanism is involved in ethyl pyruvatemediated efficacious neuroprotection in the postischemic brain, Brain Res 1060(1-2) (2005) 188-92.

60. D.R. Green, J.C. Reed, Mitochondria and apoptosis, Science 281(5381) (1998) 1309-12.

61. T. Sugawara, N. Noshita, A. Lewén, Y. Gasche, M. Ferrand-Drake, M. Fujimura, Y. Morita-Fujimura, P.H. Chan, Overexpression of Copper/Zinc Superoxide Dismutase in Transgenic Rats Protects Vulnerable Neurons against Ischemic Damage by Blocking the Mitochondrial Pathway of Caspase Activation, The Journal of Neuroscience 22(1) (2002) 209-217.

62. T. Fahmi, D. Branch, Z.A. Nima, D.S. Jang, A.V. Savenka, A.S. Biris, A.G. Basnakian, Mechanism of graphene-induced cytotoxicity: Role of endonucleases, J Appl Toxicol 37(11) (2017) 1325-1332.

63. X. Zhou, D. Patel, S. Sen, V. Shanmugam, A. Sidawy, L. Mishra, B.N. Nguyen, Poly-ADP-ribose polymerase inhibition enhances ischemic and diabetic wound healing by promoting angiogenesis, $\mathrm{J}$ Vasc Surg 65(4) (2017) 1161-1169.

64. G. Kroemer, B. Dallaporta, M. Resche-Rigon, The mitochondrial death/life regulator in apoptosis and necrosis, Annu Rev Physiol 60 (1998) 619-42.

65. S. Desagher, J.-C. Martinou, Mitochondria as the central control point of apoptosis, Trends in Cell Biology 10(9) (2000) 369-377.

66. G.H. Wang, R. Lan, X.D. Zhen, W. Zhang, J. Xiang, D.F. Cai, An-Gong-Niu-Huang Wan protects against cerebral ischemia induced apoptosis in rats: up-regulation of $\mathrm{Bcl}-2$ and down-regulation of Bax and caspase-3, J Ethnopharmacol 154(1) (2014) 156-62.

67. P. Zhao, R. Zhou, X.Y. Zhu, Y.J. Hao, N. Li, J. Wang, Y. Niu, T. Sun, Y.X. Li, J.Q. Yu, Matrine attenuates focal cerebral ischemic injury by improving antioxidant activity and inhibiting apoptosis in mice, Int $\mathrm{J}$ Mol Med 36(3) (2015) 633-44.

\section{Figures}


Neurological score

TTC staining

OXA icv.

SD rats arrived MCAO $30 \mu \mathrm{g} / \mathrm{kg}$
Immunofluorescent

Western blot
Hoechst 33342 staining ELISA

Immunofluorescent

U251 cell thawing

OGD OXA

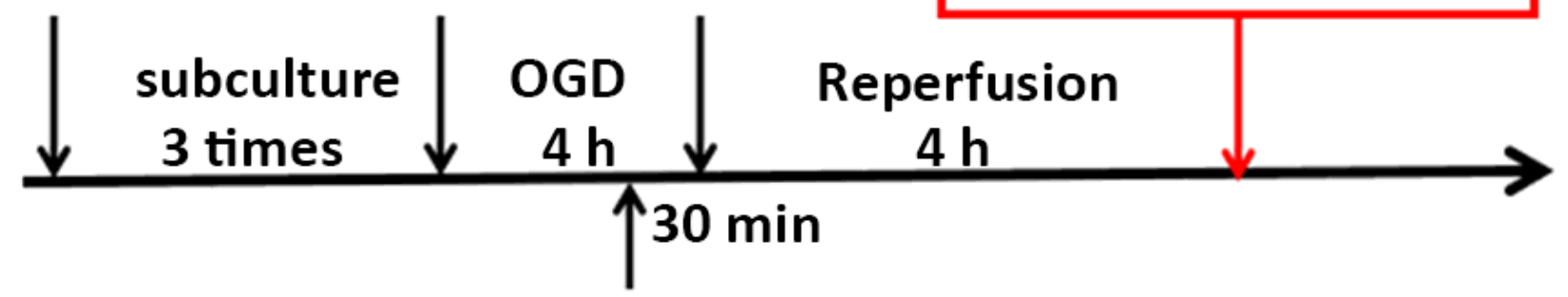

SB334867

Figure 1

Flow chart of the study design. SB334867 is an inhibitor of orexin receptor type 1 (OX1R). OXA: Orexin-A; MCAO: middle cerebral artery occlusion; TTC: 2,3,5-triphenyl-2H-tetrazolium chloride; OGD: oxygen glucose deprivation; ELISA: Cytokine Enzyme-Linked Immunosorbent Assay; SD: Sprague-Dawley. 
(A)
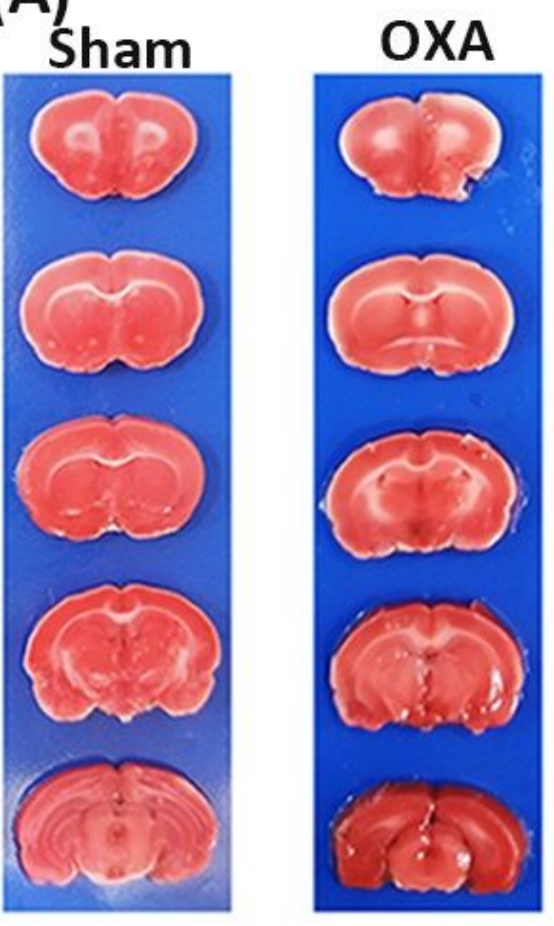

\section{MCAO/R MCAO/R+OXA}
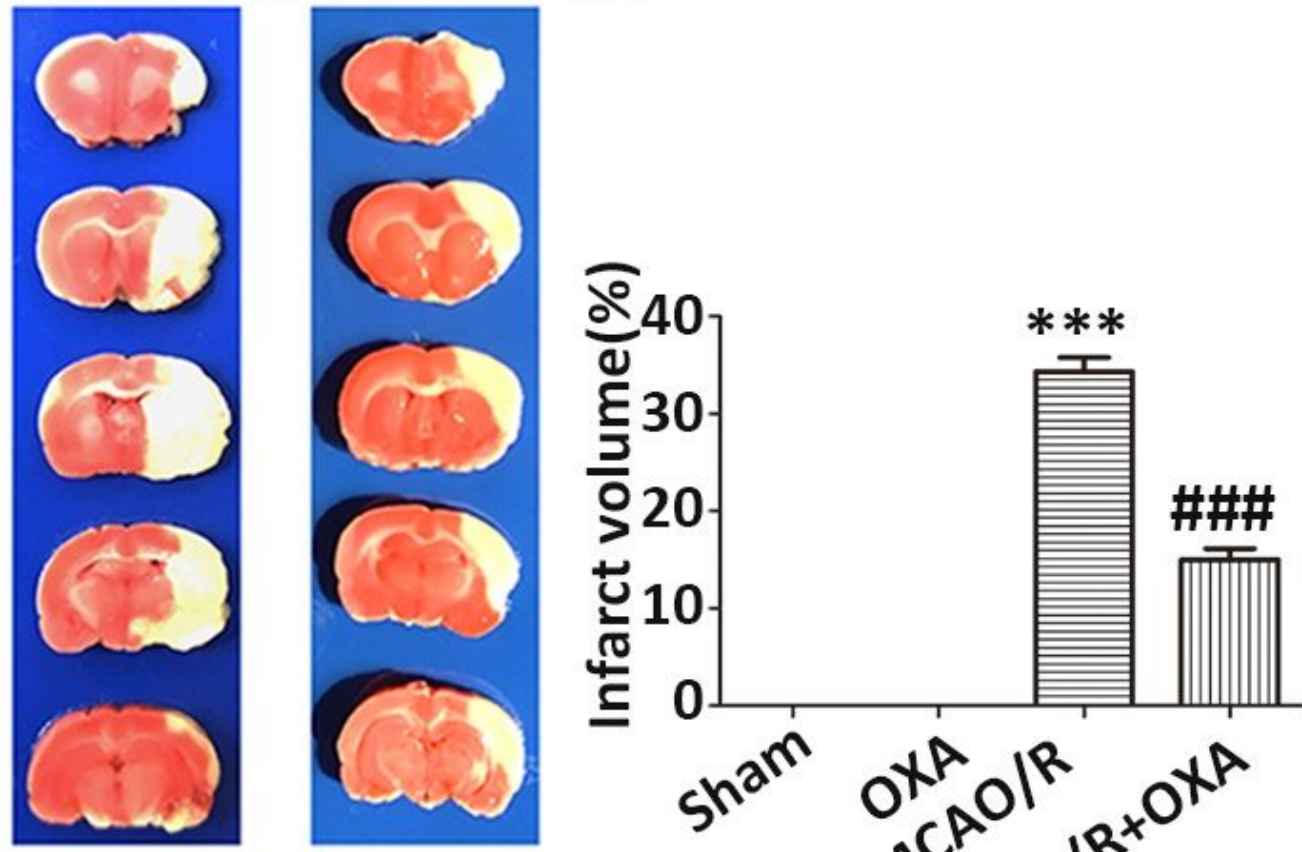

(B)

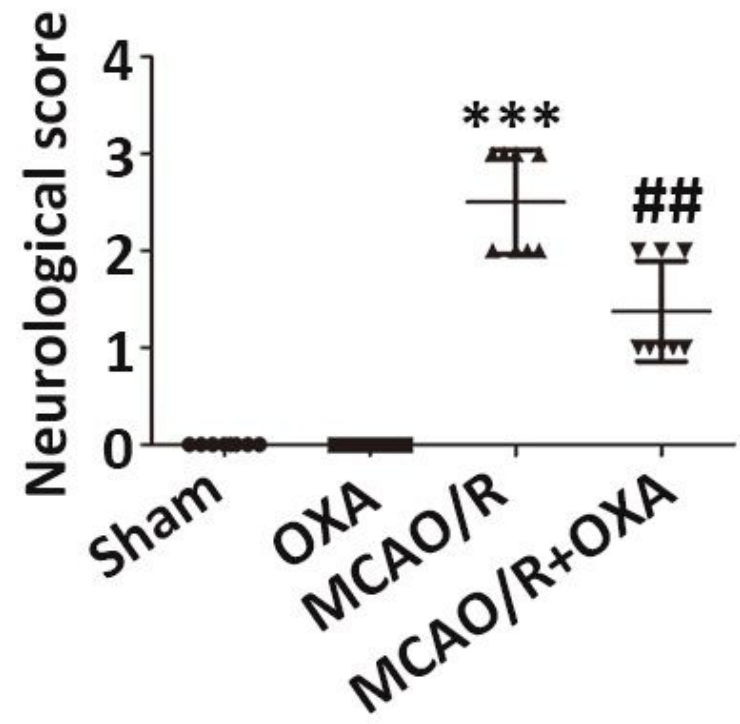

Figure 2

Neuroprotective effects of OXA on cerebral infarct volume and neurological score. (A) Representative TTC-stained coronal brain sections. The red area is normal tissue, and the white area is infarcted tissue. Treatment with OXA $(30 \mu \mathrm{g} / \mathrm{kg})$ significantly reduced infarct volume compared with MCAO/R group. (B) Neurological deficit score. ${ }^{\star \star \star} p<0.001$ vs. sham group; \#\#p<0.01 and \#\#\#p<0.001 vs. MCAO/R group. Data are represented as means \pm SEM $(n=3-10)$. MCAO/R: middle cerebral artery occlusion and reperfusion. 
(A)
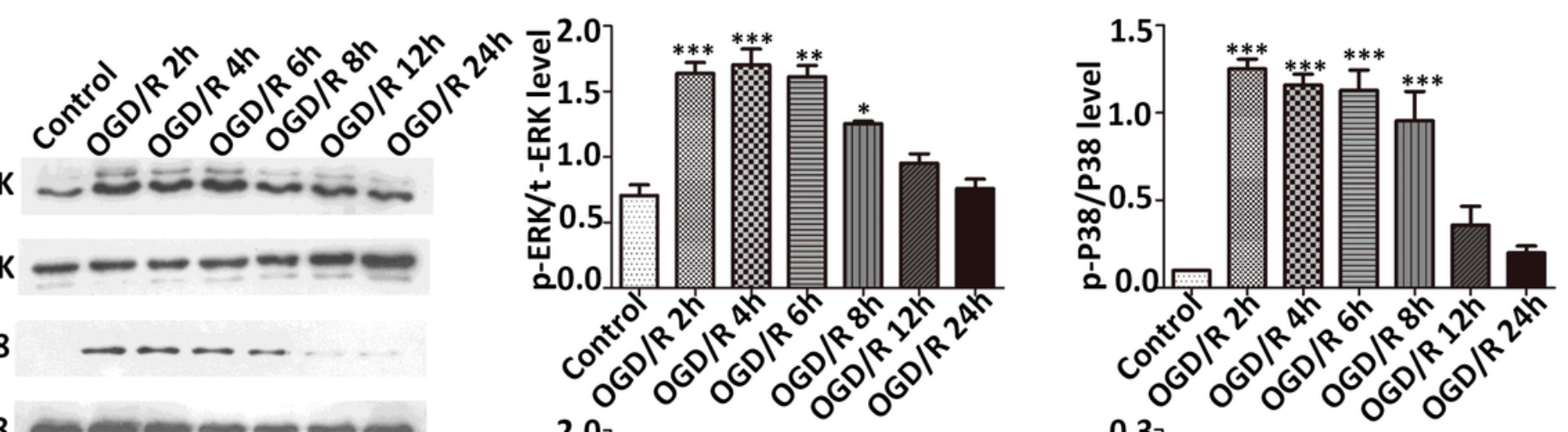

P38
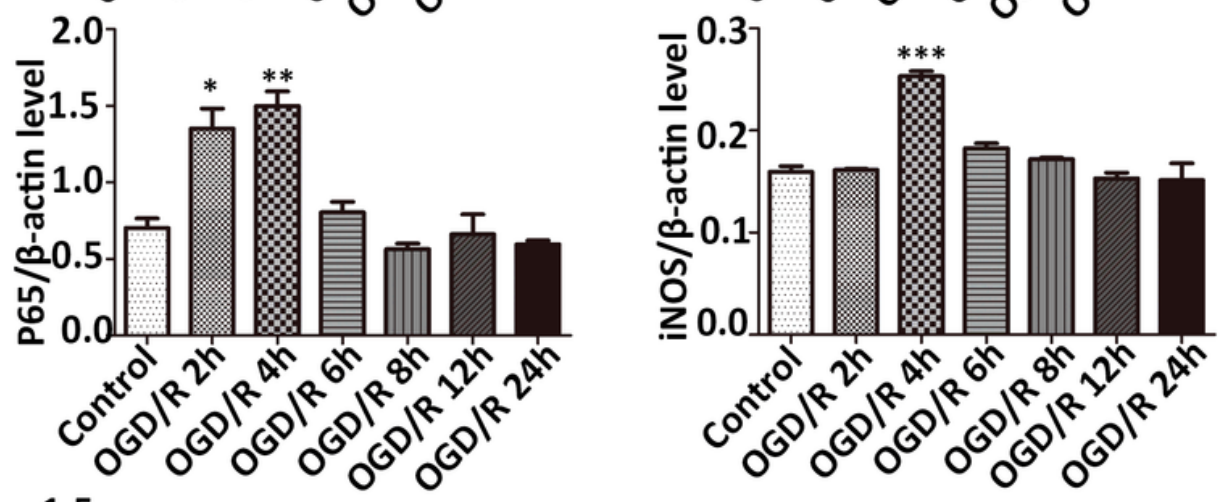

(B)

P65

iNOS

$\beta$-actin

(C)
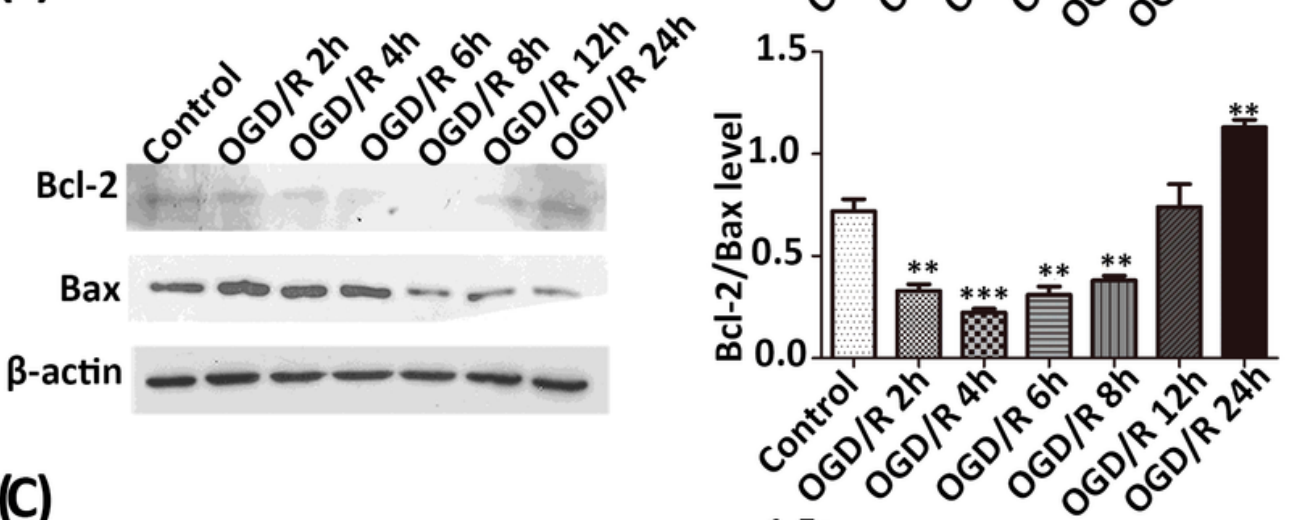

Bax

$\beta$-actin

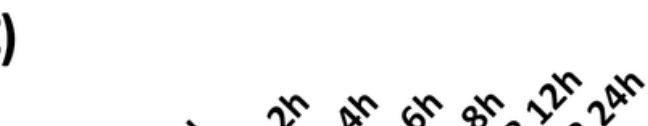

OX1R

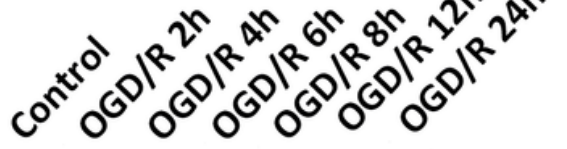

$\beta$-actin

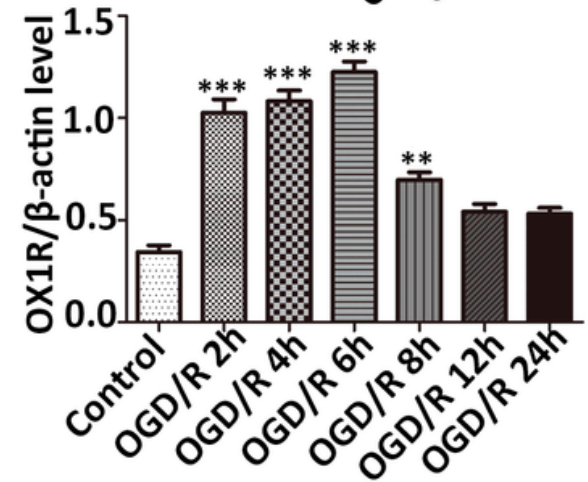

Figure 3

Levels of inflammation, apoptosis-related proteins and OX1R induced by OGD/R in U251 astrocytoma cells. Representative western blots and quantitative evaluations of the p-ERK, t-ERK, p-P38, P38, p65, iNOS (A) Bcl-2, Bax (B) and OX1R (C). ${ }^{*} p<0.05$, ${ }^{* *} p<0.01$, and ${ }^{* * *} p<0.001$ vs. sham group. Data are represented as means \pm SEM $(n=3)$. OGD/R: oxygen glucose deprivation and reperfusion. 
(A)
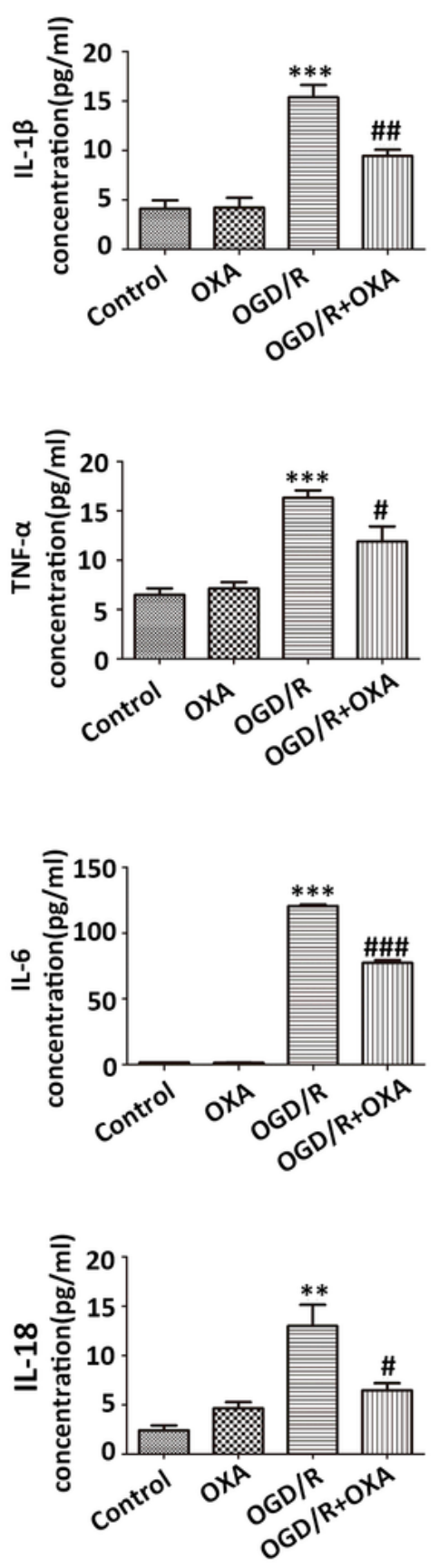

(B)

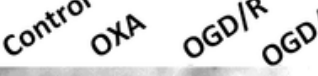

L-1 $1 \beta$

TNF- $\alpha$

IL-6

iNOS

$\beta$-actin
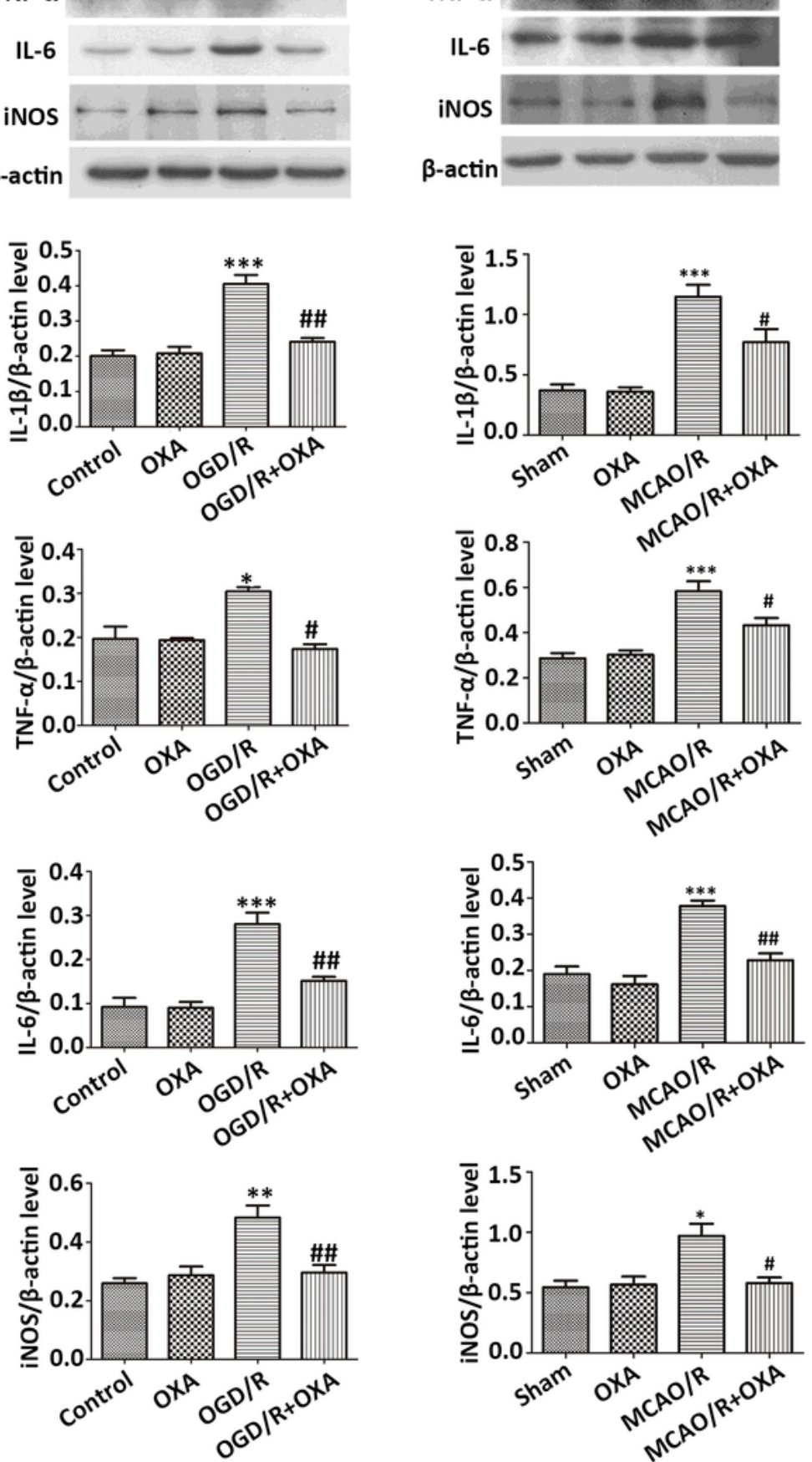

\section{Figure 4}

Effect of OXA on levels of pro-inflammatory factors in OGD/R U251 astrocytoma cells and in ischemic cortex of MCAO/R rats. (A) The concentration of pro-inflammatory factors in the supernatant of U251 astrocytoma cells and OXA significantly reduced the concentration of IL-1 $1 \beta$, TNF- $a$, IL- 6 and IL-18 in the supernatant compared with the OGD/R group. Representative western blots and quantitative evaluations of the IL-1 $\beta$, TNF-a, IL-6 and iNOS in OGD/R U251 astrocytoma cells (B) and in ischemic cortex of 
MCAO/R rats (C). Treatment with OXA significantly reduced levels of IL-1 $\beta$, TNF-a, IL-6 and iNOS in vivo and in vitro. ${ }^{*} p<0.05,{ }^{*} p<0.01$, and ${ }^{* \star *} p<0.001$ vs. control group or sham group; $\# p<0.05, \# \# p<0.01$, and \#\#\#p<0.001 vs. OGD/R group or MCAO/R group. Data are represented as means \pm SEM $(n=3)$.

(A)
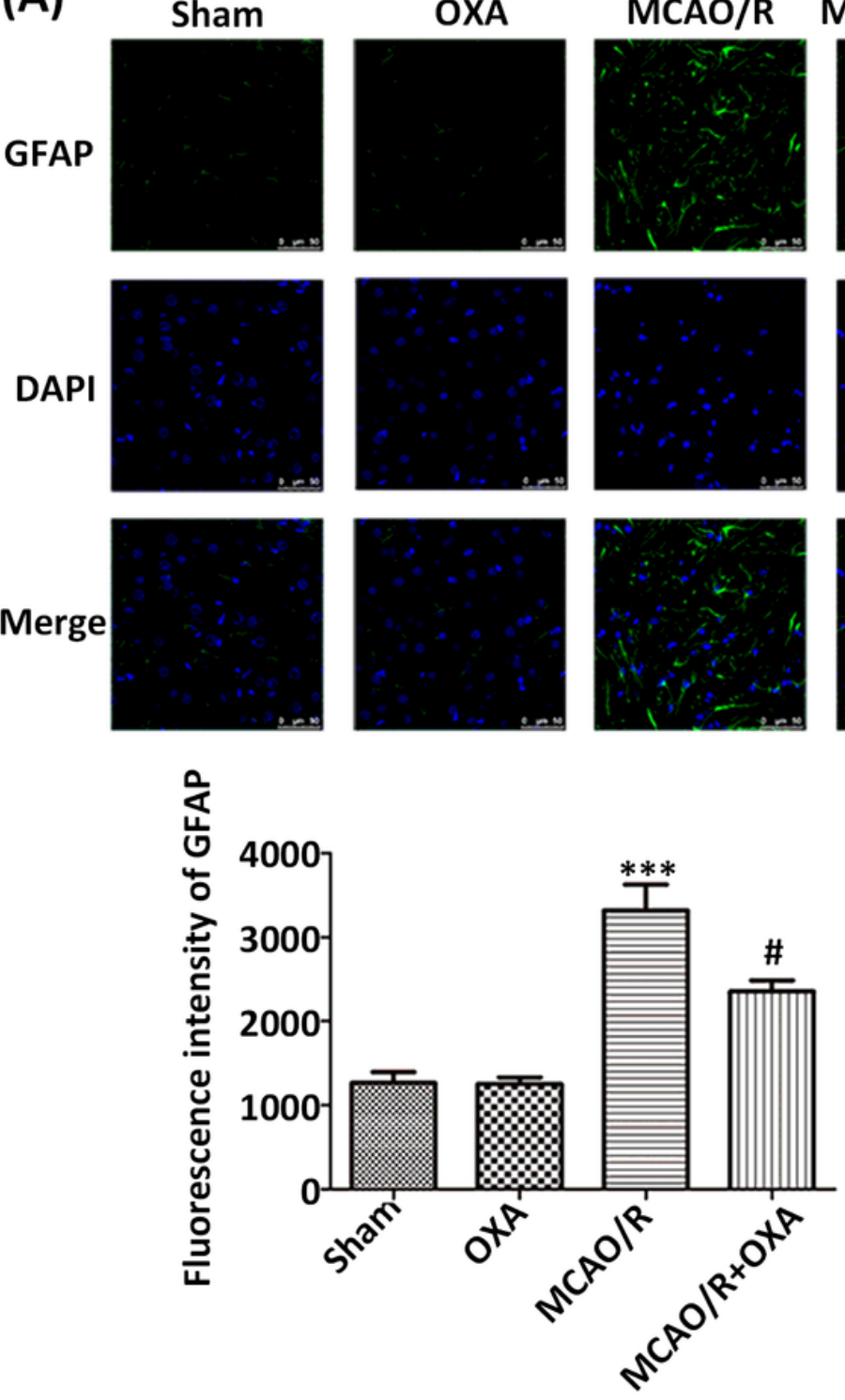

(B)
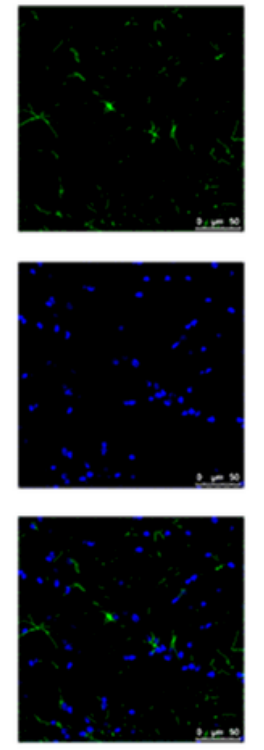

GFAP

$\beta$-actin

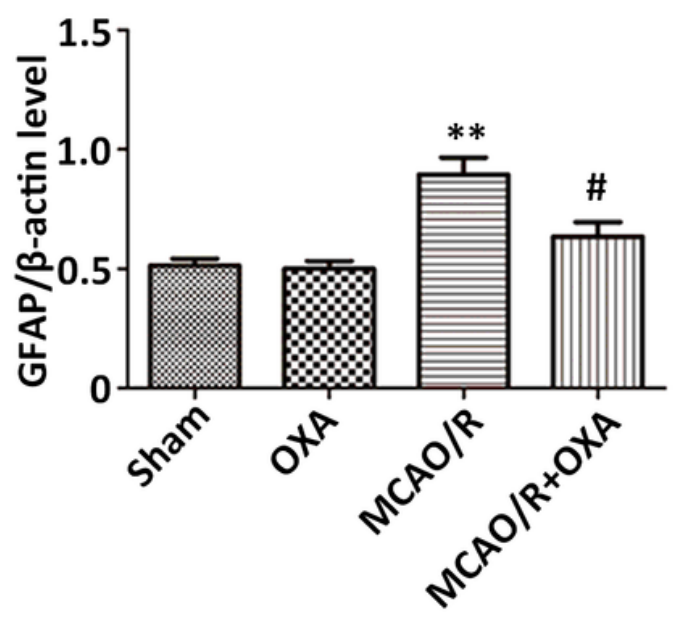

\section{Figure 5}

Effect of OXA on astrocytes activation in ischemic cortex of MCAO/R rats. (A) Immunofluorescence and quantitative analysis of GFAP. Scale bar $=50 \mu \mathrm{m}$. (B) Western blots and quantitative analyses of GFAP. OXA significantly reduced the expression of GFAP and inhibited the activation of astrocytes. ${ }^{* *} p<0.01$ and ${ }^{* \star *} p<0.001$ vs. sham group; $\# p<0.05$ vs. MCAO/R group. Data are represented as means $\pm S E M(n=3)$. GFAP: Glial fibrillary acidic protein; DAPI: 4',6-Diamidino-2-phenylindole. 
(A)

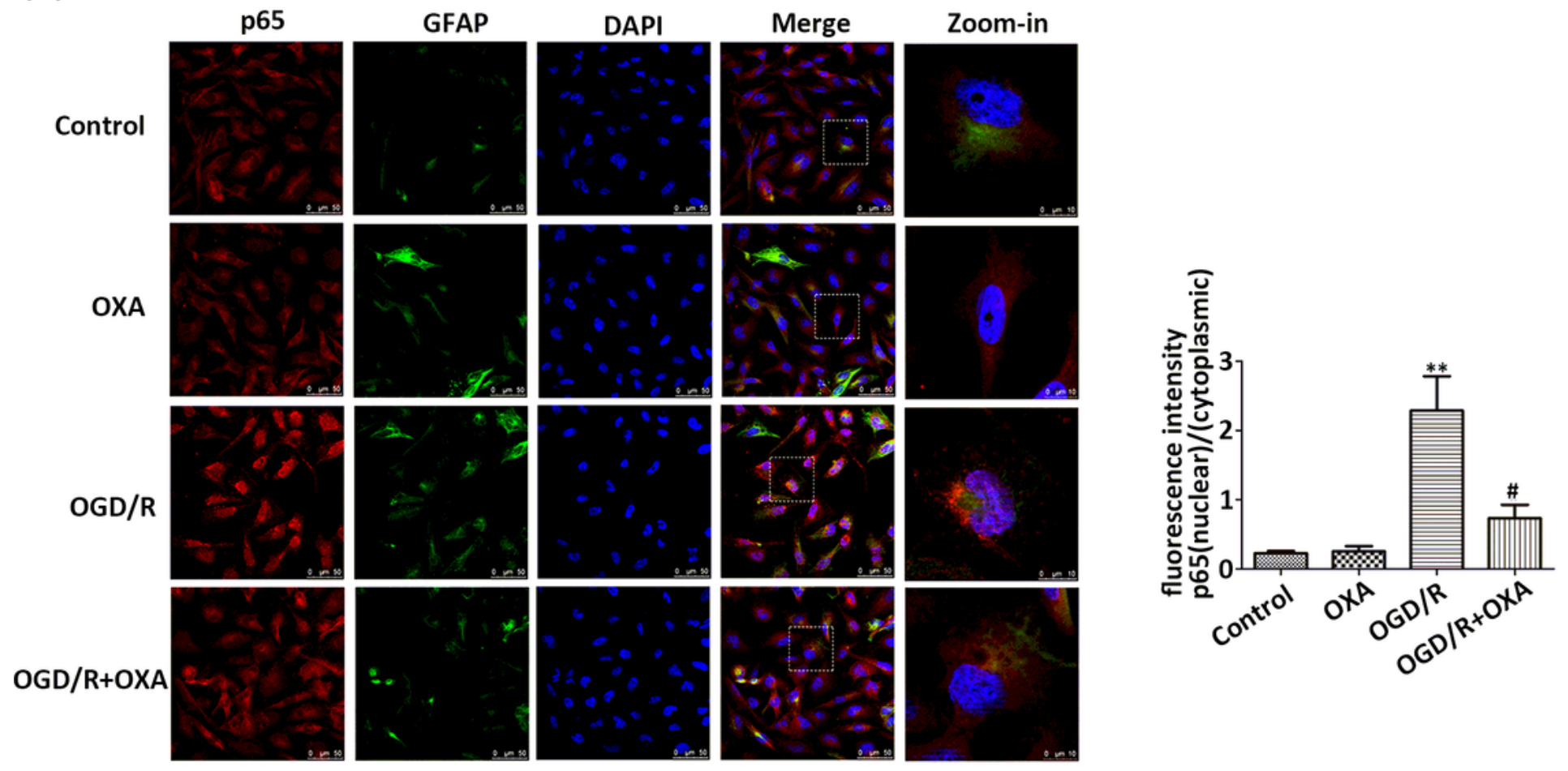

(B)
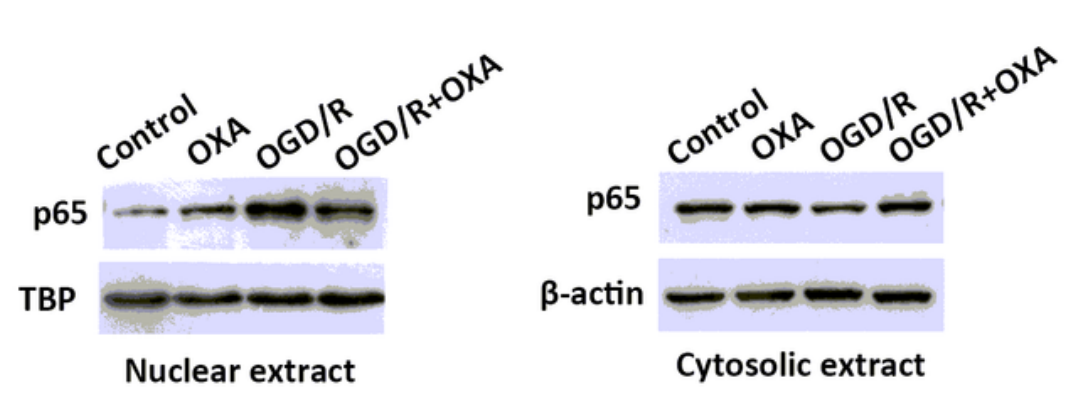

(C)

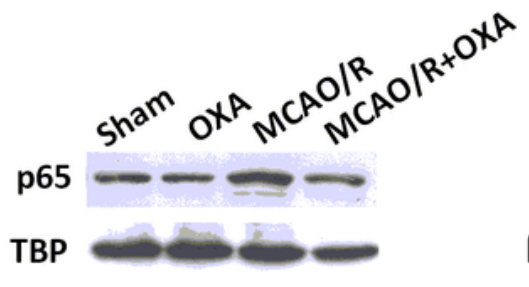

Nuclear extract

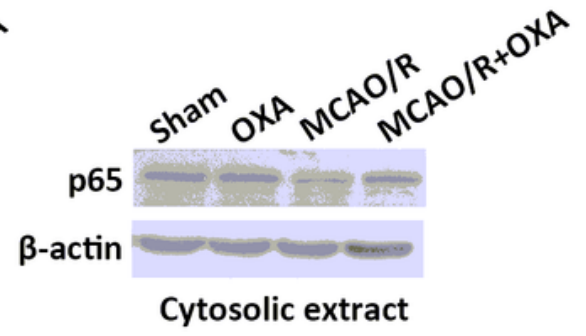

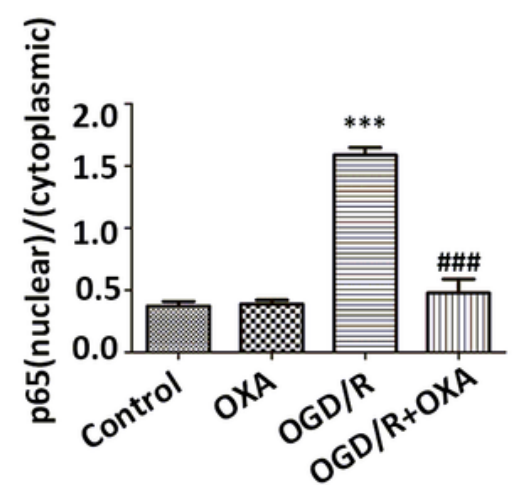

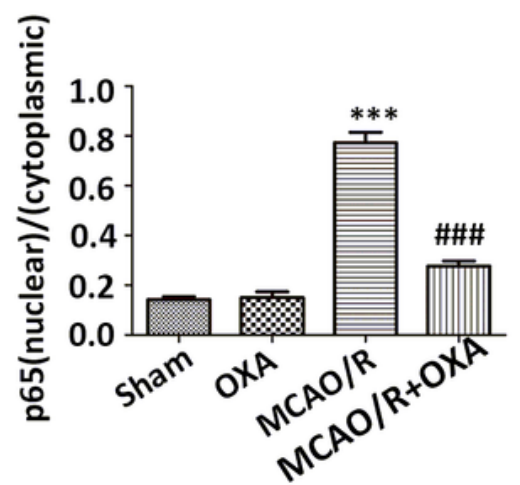

\section{Figure 6}

Effect of OXA on expression of nucleus and cytoplasm NF-KB p65 in OGD/R U251 astrocytoma cells and in ischemic cortex of MCAO/R rats. (A) Double immunofluorescence staining of p65 (red) and GFAP (green) under the intervention of OXA in OGD/R U251 astrocytoma cells. Nuclei were stained with DAPI (blue). Scale bars $=50 \mu \mathrm{m}$ or $10 \mu \mathrm{m}$. Western blot for expression of nucleus NF-kB p65 and cytoplasm NFKB p65 in OGD/R U251 astrocytoma cells (B) and ischemic cortex of MCAO/R rats (C). OXA significantly 
decreased the nuclear translocation of NF-KB p65 in vivo and in vitro. ${ }^{* \star} p<0.01$ and ${ }^{* \star *} p<0.001$ vs. control group or sham group; \#p<0.05 and \#\#\#p<0.001 vs. OGD/R group or MCAO/R group. Data are represented as means \pm SEM $(n=3)$.

(A)
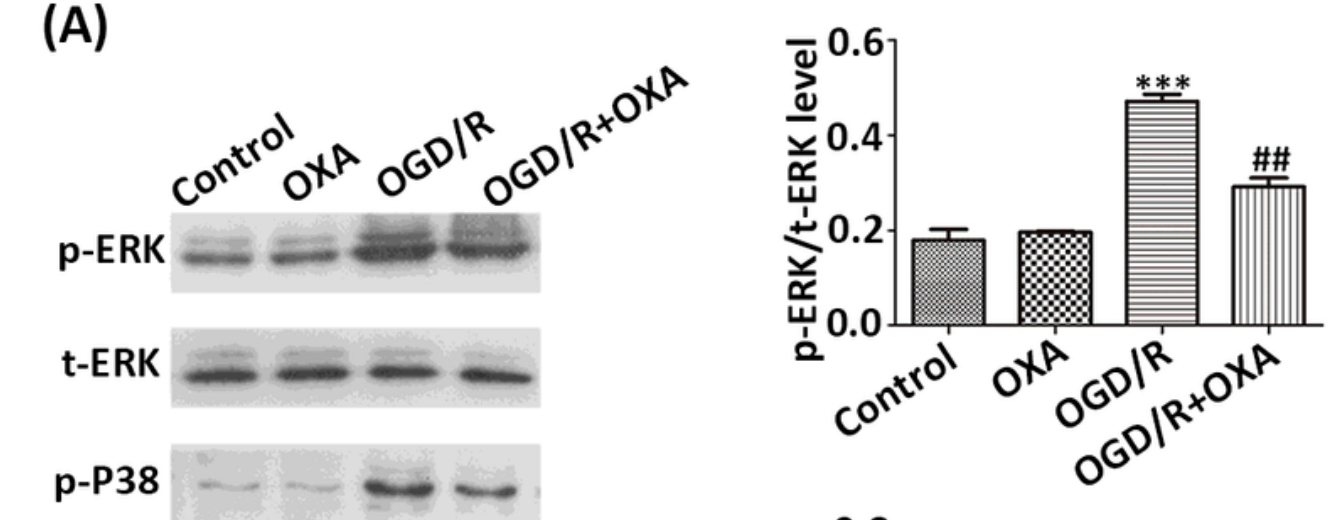

P38

$\beta$-actin

(B)
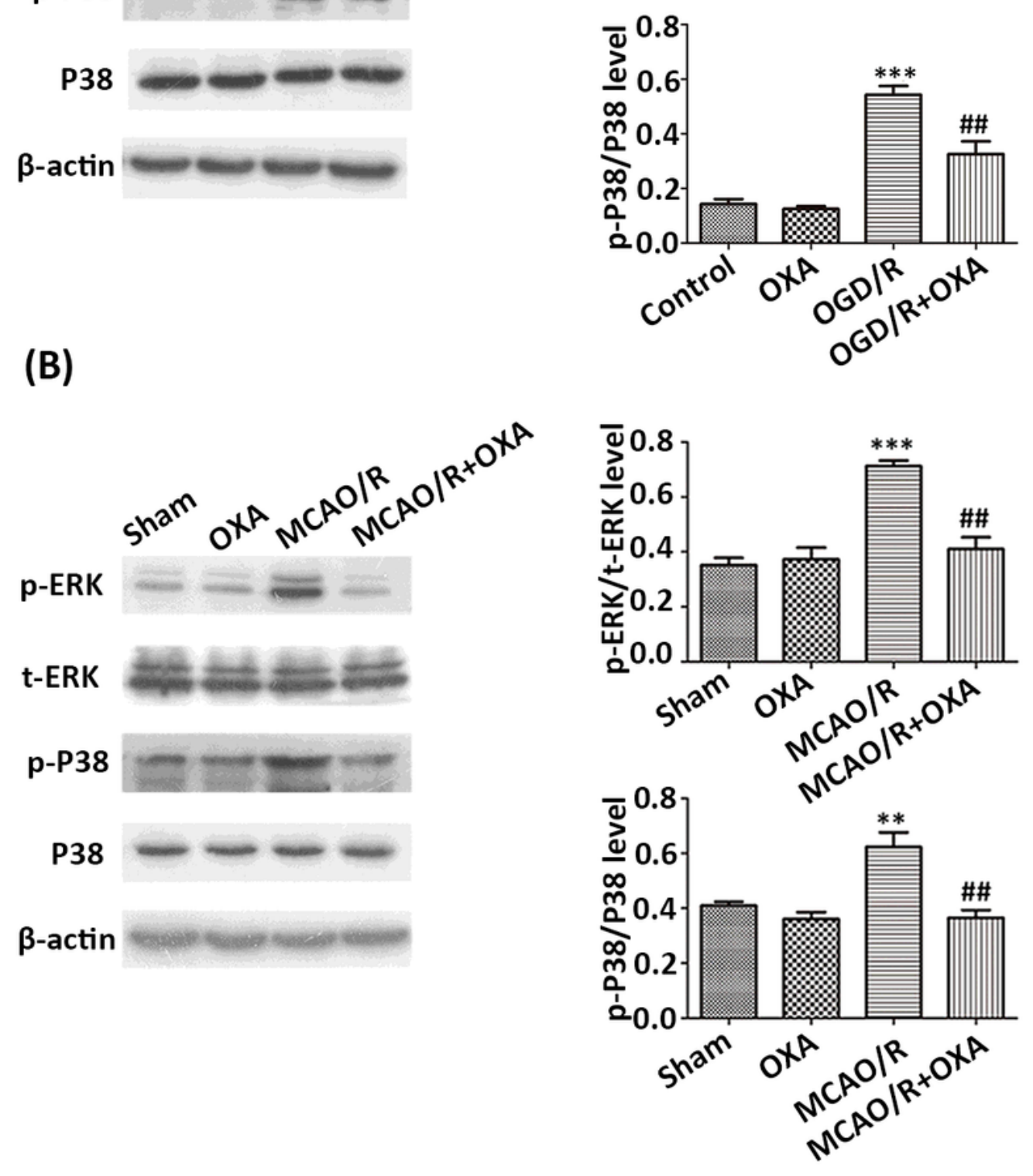

Figure 7

Effects of OXA on the expression of ERK and p38 MAPK in OGD/R U251 astrocytoma cells and in ischemic cortex of MCAO/R rats. Representative western blots and quantitative evaluations of the p-ERK, 
t-ERK, p-P38 and P38 in OGD/R U251 astrocytoma cells (A) and ischemic cortex of MCAO/R rats (B). OXA significantly inhibited the activation of ERK and p38 MAPK in vivo and in vitro. ${ }^{*} p<0.01$ and ${ }^{* \star *} p<0.001$ vs. control group or sham group; \#\#p<0.01 vs. $\mathrm{OGD} / \mathrm{R}$ group or MCAO/R group. Data are represented as means $\pm \operatorname{SEM}(n=3)$.

(A)

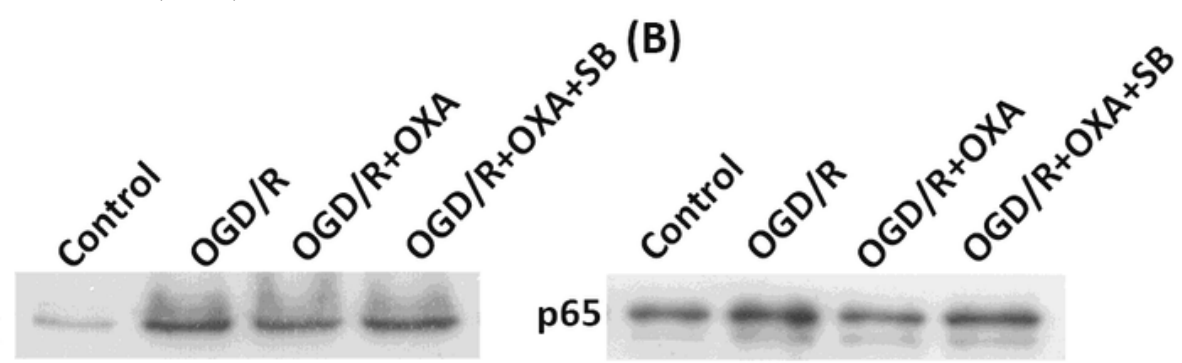

t-ERK

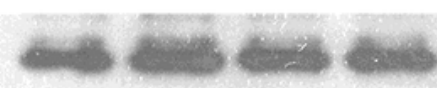

TBP

p-P38

Nuclear extract

P38

$\beta$-actin
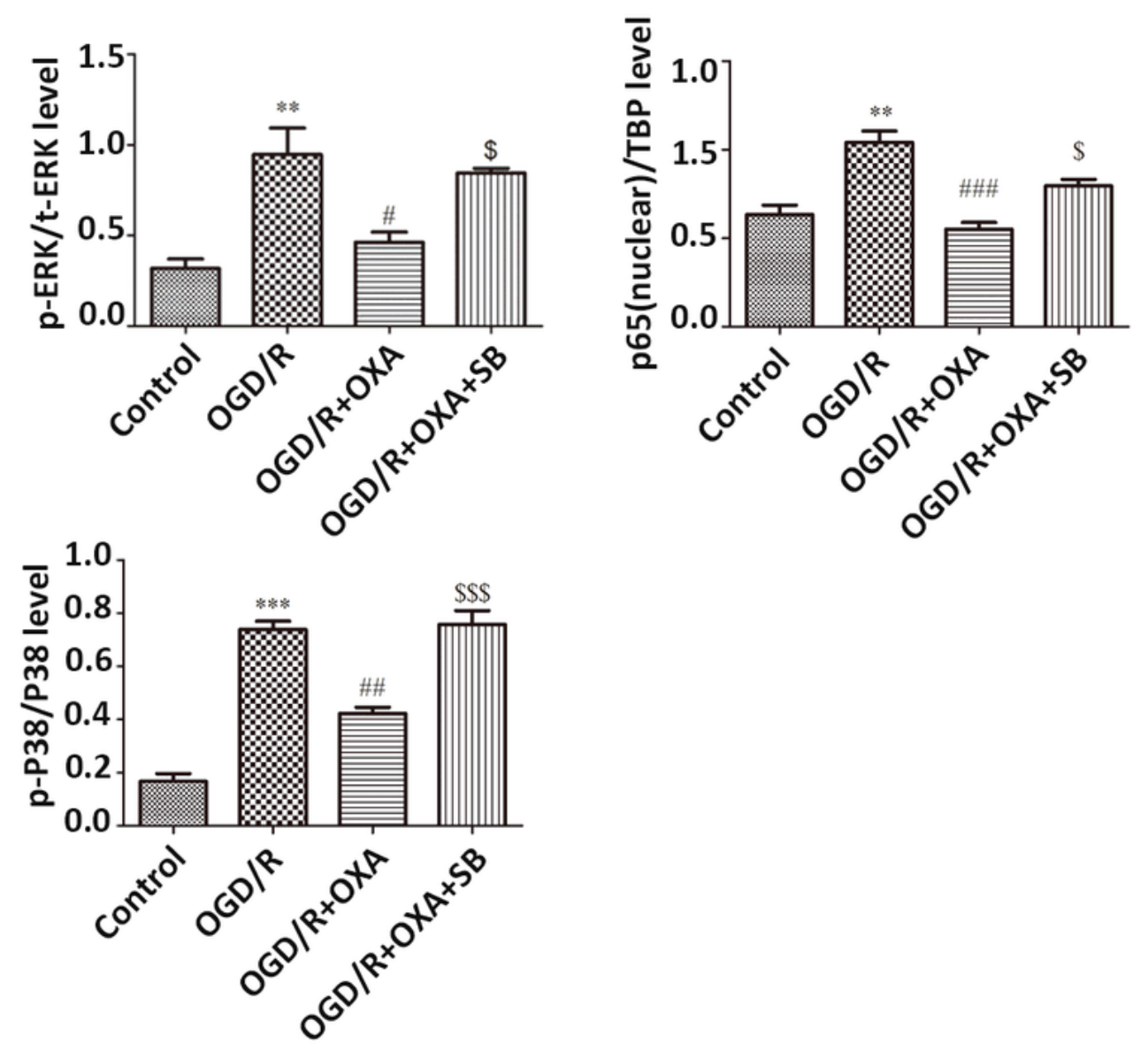

Figure 8 
Effect of OXA on NF-KB p65 and MAPK pathways under the joint intervention of OXA and SB334867, OXIR antagonist. Representative western blots and quantitative evaluations of the $p$-ERK, t-ERK, p-P38, P38 (A) and p65 in the nucleus (B). SB334867 reversed the inhibitory effect of OXA on NF-KB p65, ERK and p38 MAPK. ${ }^{* \star} p<0.01$ and ${ }^{* \star *} p<0.001$ vs. control group; $\# p<0.05$, $\# \# p<0.01$, and $\# \# \# p<0.001$ vs. OGD/R group; $\$ p<0.05$ and $\$ \$ \$ p<0.001$ vs. OGD/R+OXA group. Data are represented as means $\pm S E M$ $(n=3)$. SB: SB334867.

(A)
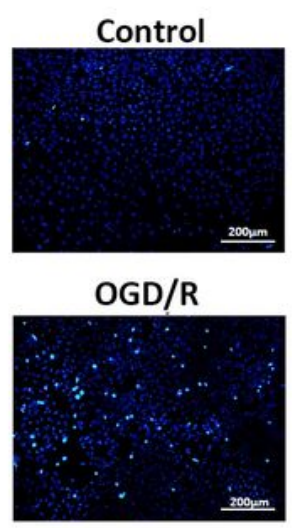

(B)

Bcl-2

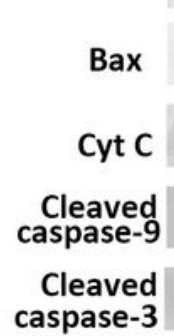

$\beta$-actin

(C)

Bcl-2

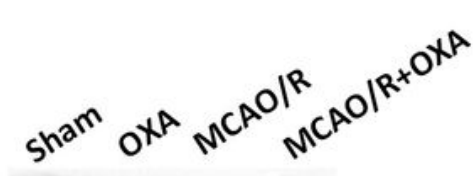

Bax

Cyt C Cleaved
caspase-9

Cleaved caspase-3

$\beta$-actin
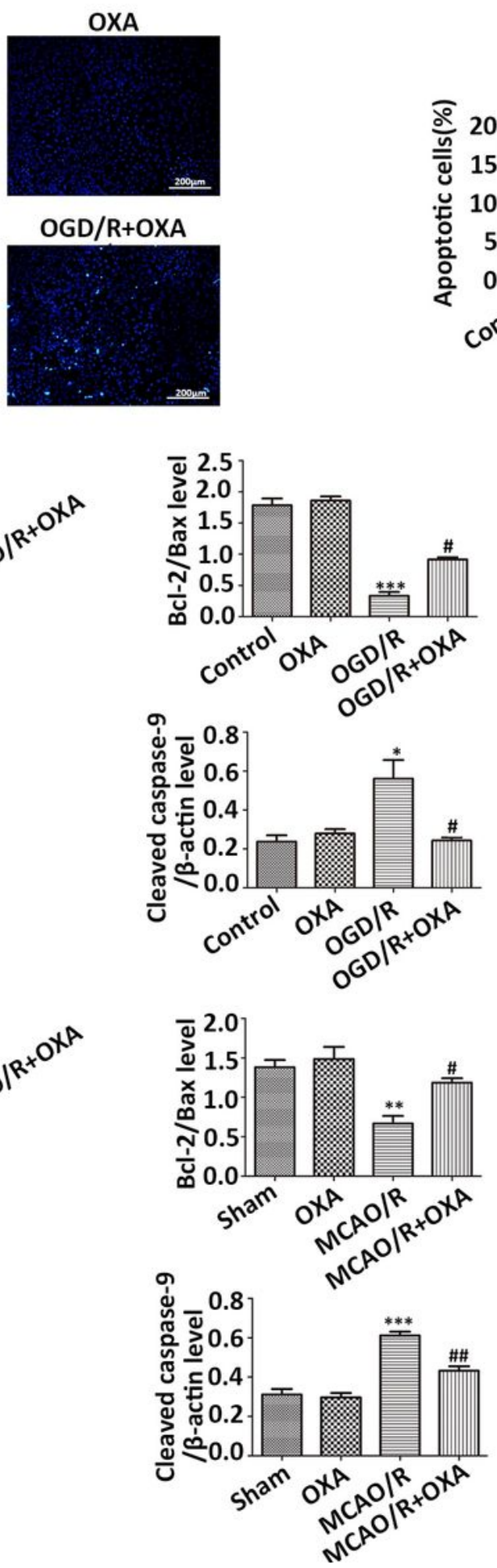
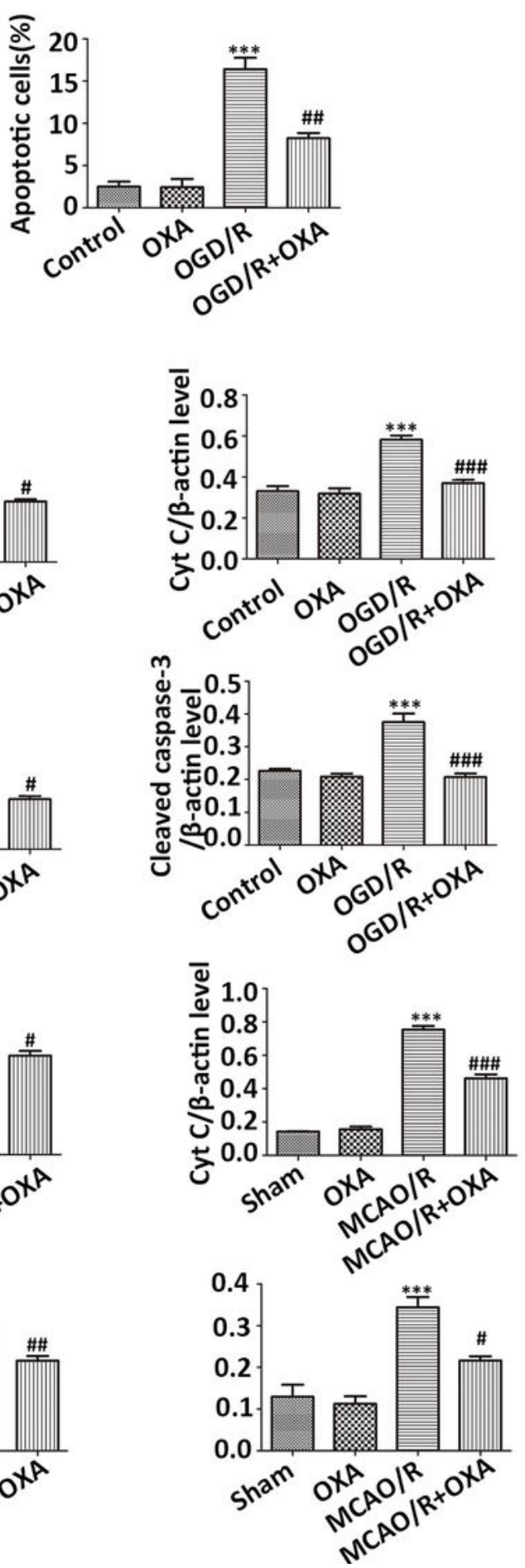

Figure 9 
Effect of OXA on apoptosis in OGD/R U251 astrocytoma cells and in the ischemic cortex of MCAO/R rats. (A) Apoptosis of U251 astrocytoma cells detected by Hoechst 33342 staining (blue). The apoptotic cell ratio was significantly reduced by OXA treatment. Scale bars $=200 \mu \mathrm{m}$. Representative western blots and quantitative evaluations of the Bcl-2, Bax, Cyt C, cleaved caspase-9 and cleaved caspase-3 in OGD/R U251 astrocytoma cells (B) and in the ischemic cortex of MCAO/R rats (C). ${ }^{*} p<0.05,{ }^{* *} p<0.01$, and

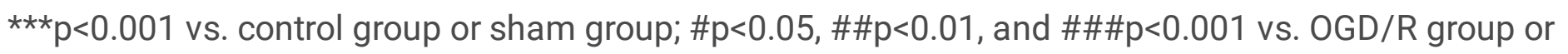
MCAO/R group. Data are represented as means \pm SEM $(n=3)$.

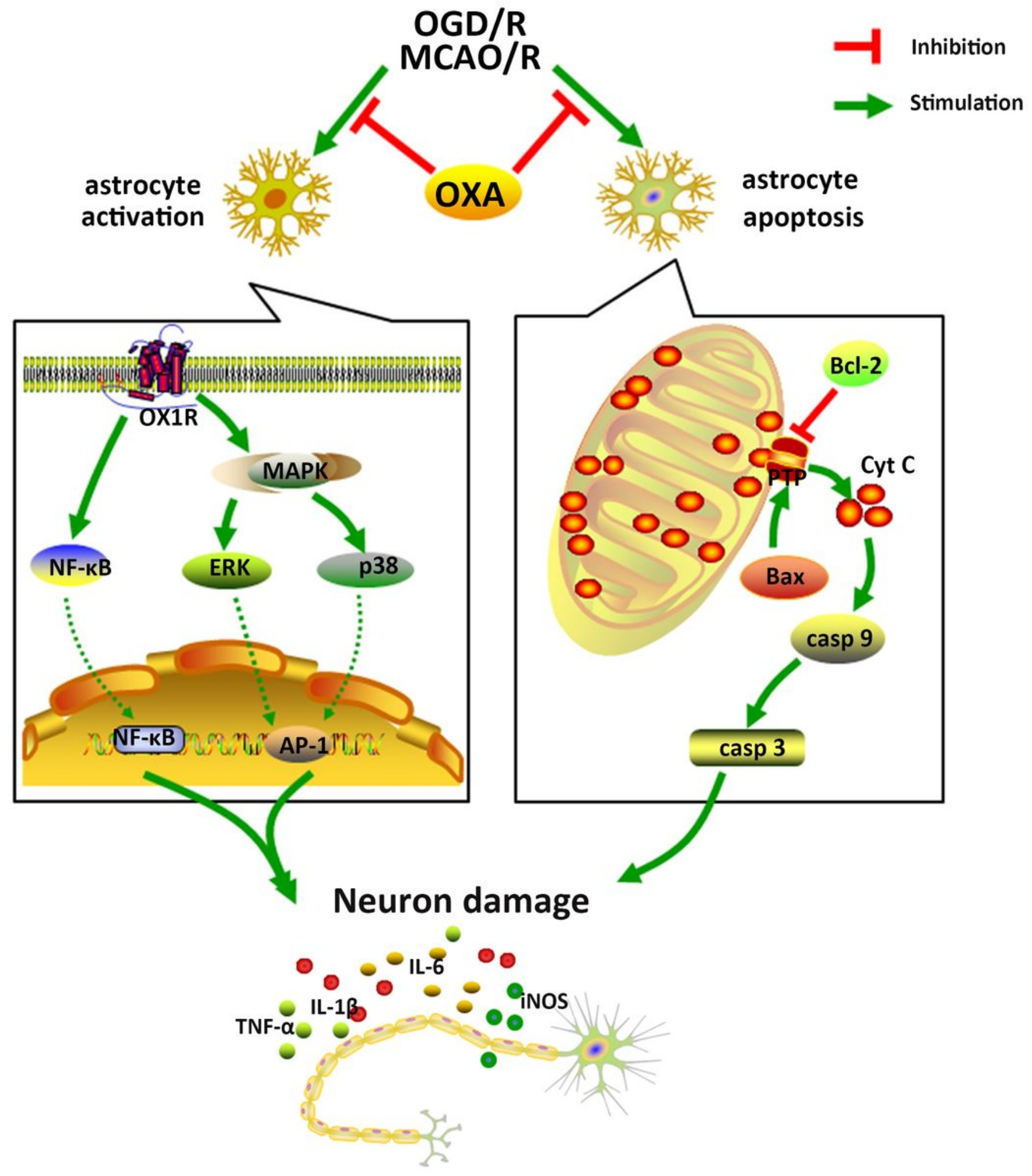




\section{Figure 10}

Schematic diagram of the mechanism of OXA inhibiting inflammation and apoptosis. The activation and apoptosis of astrocytes and inflammation are involved in CIRI. OXA inhibits these processes to reduce CIRI. OXA inhibits the phosphorylation of ERK and p38 in the MAPK pathway, thereby preventing the downstream protein AP-1 from being transported to the nucleus. OXA also inhibits the nuclear translocation of NF-kB p65. Finally, the reduction of pro-inflammatory factors reduces neuronal damage due to the anti-inflammatory effects of OXA. The opening of PTP results in the release of Cyt $C$ from the mitochondria into the cytoplasm, while the opening of PTP by Bcl-2 and Bax is weakened and enhanced, respectively. Then, Cyt $C$ activates caspase- 9 , which in turn activates caspase- 3 , and ultimately leads to cell apoptosis. However, OXA can attenuate the activation of this apoptotic pathway and reduce the apoptosis of astrocytes and other nerve cells. OXA: Orexin-A; OGD/R: oxygen glucose deprivation and reperfusion; MCAO/R: middle cerebral artery occlusion and reperfusion; OX1R: orexin receptor type 1; NFKB: Nuclear factor kappa light-chain enhancer of activated B cells; MAPK: mitogen-activated protein kinase; ERK: extracellular signal-regulated kinase; PTP: permeability transition pores. 